\title{
Pulsar microstructure and its quasi-periodicities with the S2 VLBI system at a resolution of 62.5 nanoseconds
}

\author{
M. V. Popov ${ }^{1}$, N. Bartel ${ }^{2}$, W. H. Cannon ${ }^{2,3}$, A. Yu. Novikov ${ }^{3}$, V. I. Kondratiev ${ }^{1}$, and V. I. Altunin ${ }^{4}$ \\ 1 Astro Space Center of the Lebedev Physical Institute, Profsoyuznaya 84/32, Moscow 117997, Russia \\ 2 York University, Department of Physics and Astronomy, 4700 Keele Street, Toronto, Ontario, M3J 1P3, Canada \\ 3 Space Geodynamics Laboratory/CRESTech, 4850 Keele Street, Toronto, Ontario M3J 3K1, Canada \\ 4 Jet Propulsion Laboratory, 4800 Oak Grove Drive, Pasadena, CA 91109, USA
}

Received 5 April 2001 / Accepted 19 September 2002

\begin{abstract}
We report a study of microstructure and its quasi-periodicities of three pulsars at $1.65 \mathrm{GHz}$ with the S2 VLBI system at a resolution of $62.5 \mathrm{~ns}$, by far the highest for any such statistical study yet. For PSR B1929+10 we found in the average cross-correlation function (CCF) broad microstructure with a characteristic timescale of $95 \pm 10 \mu$ s and confirmed microstructure with characteristic timescales between 100 and $450 \mu$ s for PSRs B0950+08 and B1133+16. On a finer scale PSRs B0950+08, B1133+16 (component II) and B1929+10 show narrow microstructure with a characteristic timescale in the $\mathrm{CCFs}$ of $\sim 10 \mu \mathrm{s}$, the shortest found in the average CCF or autocorrelation function (ACF) for any pulsar, apart perhaps for the Crab pulsar. Histograms of microstructure widths are skewed heavily toward shorter timescales but display a sharp cutoff. The shortest micropulses have widths between 2 and $7 \mu \mathrm{s}$. There is some indication that the timescales of the broad, narrow, and shortest micropulses are, at least partly, related to the widths of the components of the integrated profiles and the subpulse widths. If the shortest micropulses observed are indeed due to beaming then the ratio, $\gamma$, of the relativistic energy of the emitting particles to the rest energy is about 20000 , independent of the pulsar period. We predict an observable lower limit for the width of micropulses from these pulsars at $1.65 \mathrm{GHz}$ of $0.5 \mu \mathrm{s}$. If the short micropulses are instead interpreted as a radial modulation of the radiation pattern, then the associated emitting sources have dimensions of about $3 \mathrm{~km}$ in the observer's frame. For PSRs B0950+08 and B1133+16 (both components) the micropulses had a residual dispersion delay over a 16-MHz frequency difference of $\sim 2 \mu \mathrm{s}$ when compared to that of average pulse profiles over a much larger relative and absolute frequency range. This residual delay is likely the result of propagation effects in the pulsar magnetosphere that contribute to limiting the width of micropulses. No nanopulses or unresolved pulse spikes were detected. Cross-power spectra of single pulses show a large range of complexity with single spectral features representing classic quasi-periodicities and broad and overlapping features with essentially no periodicities at all. Significant differences were found for the two components of PSR B1133+16 in every aspect of our statistical analysis of micropulses and their quasi-periodicities. Asymmetries in the magnetosphere and the hollow cone of emission above the polar cap of the neutron star may be responsible for these differences.
\end{abstract}

Key words. stars: pulsars: general - radio continuum: stars - methods: data analysis - methods: observational

\section{Introduction}

Pulsar radio emission originates in a region of extremely small size, most likely from charged particles in the magnetosphere traveling along the diverging dipole magnetic field lines above the polar cap of a neutron star (e.g., Ruderman \& Sutherland 1975; Arons 1983). This emission can be seen by an external observer only during short successive time windows separated by the neutron star's rotation period. Within such a window, the radio signals recorded at different times are therefore related to different reference points in the pulsar magnetosphere which are either separated longitudinally across the beam or radially along the beam or by a combination of both. Correspondingly,

Send offprint requests to: M. V. Popov, e-mail: mpopov@asc.rssi.ru the observed intensity fluctuations can be either caused by a longitudinal modulation of the radiation pattern over the cross section of the polar magnetic field lines or by a radial modulation of the radiation pattern along the opening polar magnetic field lines, or, again, by a combination of both. The longitudinal modulation is most likely related to the stationary geometry of the emission beam fixed to each of the poles of the rotating neutron star. The radial modulation is likely related to plasma bunching and linked to the elementary emission mechanism. In this model the spectrum of the radio emission is a function of the radial distance from the neutron star, and the beam width is frequency dependent. High frequency radiation is emitted closer to the neutron star and the beam is narrower, low frequency radiation is emitted further out and the beam is broader, reflecting the opening of the polar magnetic field 
lines. The study of pulsar intensity fluctuations has largely the goal of probing on the one hand the geometrical characteristics of the pulsar emission beam and its underlying magnetospheric structure and on the other hand the elementary emission mechanism.

Pulsar radio emission is known to exhibit fluctuations over a broad range of timescales. Average pulse profiles can have up to seven components (Kramer 1994) and together with their frequency dependent widths reflect best the underlying geometrical structure of the magnetosphere (Rankin 1983). Every individual pulse is composed of one or several separate subpulses. In general, the subpulses fluctuate strongly within a single pulse and from pulse to pulse but have stationary characteristics and characteristic widths computed from their autocorrelation functions that are $96 \%$ correlated with the width of the strongest component of the average pulse profile (Bartel et al. 1980). Like the average pulse profiles they most likely also reflect the geometrical structure of the magnetosphere (Bartel et al. 1980). The subpulses in their turn are often composed of micropulses or microstructure with typical timescales of about hundred to a few hundred microseconds, or several tenths of a degree in pulsar longitude (e.g., Hankins 1972; Kardashev et al. 1978). In a few cases still much faster but well resolved individual fluctuations were recorded, for instance with a timescale down to $2.5 \mu$ s for PSR B1133+16 (Bartel \& Hankins 1982, see also Bartel 1978), the fastest fluctuations found for any pulsar apart from the Crab pulsar. For the latter pulsar sporadic giant pulses were observed which were still unresolved at a time resolution of $10 \mathrm{~ns}$ (Hankins 2000).

For the broader micropulses, quasi-periodic structures were found in the very first studies of single pulses with sufficiently high time resolution. Hankins (1971) found many examples of regularly spaced micropulses with periods of 300 to $700 \mu$ s for PSR B0950+08 at a frequency of $111.5 \mathrm{MHz}$. Backer (1973), Boriakoff (1976) and Cordes (1976a) have shown that PSR B2016+28 has quasi-periodic microstructure with periods ranging from 0.6 to $1.1 \mathrm{~ms}$ at a frequency of $430 \mathrm{MHz}$. Soglasnov et al. $(1981,1983)$ analyzed the statistics of quasi-periodicities for PSRs B0809+74 and B1133+16 at $102.5 \mathrm{MHz}$. Cordes et al. (1990) studied five pulsars (including PSRs B0950+08 and B1133+16) with quasi-periodic microstructure at several radio frequencies. They concluded that there are no preferred periods for quasi-periodicities that are intrinsic to a given pulsar and that there is no frequency dependence of the micropulse width and the characteristic period of the quasi-periodicity.

Lange et al. (1998) studied seven bright pulsars, including our three, at 1.41 and $4.85 \mathrm{GHz}$ with a time resolution between $7 \mu \mathrm{s}$ and $160 \mu \mathrm{s}$. They did not find notable differences of microstructure parameters at different frequencies.

It is the topic of this paper to investigate micropulses and their quasi-periodicities and to help to understand whether they reflect the longitudinally modulated emission pattern and the geometry of the magnetosphere (e.g., Benford 1977) or instead are more effected by the radially or intrinsically temporally modulated pulsar emission pattern (e.g., Hankins 1972; Cordes 1981). The shortest micropulses observable in a pulsar in particular may harbor essential clues about the nature of the emission fluctuations in pulsars.

To achieve a high time resolution one must digitally record the pulsar signal before detection with subsequent dispersion removal processing as originally described by Hankins (1971). Previous studies were based mainly on observations made with a time resolution of several microseconds to several tens of microseconds. A review of microstructure research is given by Hankins (1996). In this paper we present a statistical analysis of the properties of microstructure for PSRs B0950+08, $\mathrm{B} 1133+16$, and B1929+10 at $1650 \mathrm{MHz}$ with a time resolution of $62.5 \mathrm{~ns}$, the most extensive such analysis yet for any pulsar and with one of the highest time resolutions ever used.

\section{Observations}

The observations were made with the NASA Deep Space Network 70-m DSS43 radio telescope at Tidbinbilla, Australia. PSRs B0950+08 and B1133+16 were observed on 10 May 2000 and PSR B1929+10 on 24 April 1998.

The data were recorded continuously with the S2 VLBI system (Cannon et al. 1997; Wietfeldt et al. 1998) in the 2-bit sampling mode in the lower sideband from 1634 to $1650 \mathrm{MHz}$ and the upper sideband from 1650 to $1666 \mathrm{MHz}$. Left circular polarization was recorded for both frequency channels. The observations were made in absentia which is more typical for VLBI observations. In general, pulsar observations with the S2 VLBI system can be made at any of the $\sim 30$ radio telescopes worldwide which are equipped with such a system, in the same way VLBI observations are made without the need for the investigator's presence. In effect, a dedicated pulsar backend at the observing station is replaced with a software package on the workstation at the investigator's home institution.

\section{Data reduction}

The tapes were shipped to Toronto and played back through the S2 Tape-to-Computer Interface (S2-TCI) at the Space Geodynamics Laboratory (SGL) of CRESTech on the campus of York University. The S2-TCI system transfers the basebandsampled pulsar data to computer files stored on hard disks and makes the data available for off-line analysis by a SUN Workstation computer. A similar use of the S2-TCI system for processing observations of PSR J0437-4715 was reported by Kempner et al. (1997).

The S2-TCI system enabled us to transfer the data stream to disk in a piecewise manner, the size or duration of each piece of data stream being limited by the disk storage capacity. In general, we transferred a set of about $10 \mathrm{~min}$ of data to disk at a time. In a first step we detected the recorded signal by squaring and averaging it with a time constant of about $100 \mu \mathrm{s}$. Then we determined the phase of the ON-pulse window and selected strong pulses. For the selection we computed the signal-to-noise ratio, $S N R=\frac{\left\langle I_{\mathrm{on}}\right\rangle}{\left\langle I_{\mathrm{of}}\right\rangle}$, where $\left\langle I_{\mathrm{on}}\right\rangle$ is the mean intensity in the ON-pulse window after subtraction of the mean intensity in the OFF-pulse window, $\left\langle I_{\text {off }}\right\rangle$. The $S N R$ so defined corresponds to the relative increase of the antenna temperature in the ON-pulse window and is therefore relatively small even 
Table 1. Pulsar characteristics and fixed parameters: $P$ is the pulsar period; $D M$ is the dispersion measure for a dispersion constant: $\alpha_{\mathrm{d}}=$ $2.41 \times 10^{-16} \mathrm{~cm}^{-3} \mathrm{pc} \mathrm{s} ; \delta t_{\text {cal }}$ is the computed time delay between the two frequency channels based on $D M$, the standard errors are $<0.1 \mu \mathrm{s}$; $N$ is the approximate total number of pulsar periods observed; $N_{\mathrm{p}}$ is the number of selected strong pulses used to compute the CCFs. For PSR B1929+10 a distinction is made between the number used to compute the average CCF and the number (in parentheses) used to compute the individual CCFs for the analysis of individual pulses; $N_{\mu}$ is the number of microstructure features revealed; $N_{\mu-Q P}$ is the number of detected quasi-periodicities; $N_{\text {no- } \mu}$ is the number of smooth structureless pulses; $T$ is the number of samples for the duration of the pulse window used to calculate the CCF; SNR is the signal-to-noise ratio of the mean pulse intensity in the ON-pulse window averaged over all pulses selected for processing. It is a measure of the relative increase of the antenna temperature in the ON-pulse window. For the specific definition of $S N R$, see text.

\begin{tabular}{lccrccccccc}
\hline \hline PSR B & $\begin{array}{c}P \\
(\mathrm{~s})\end{array}$ & $\begin{array}{c}D M \\
\left(\mathrm{pc} / \mathrm{cm}^{3}\right)\end{array}$ & \multicolumn{1}{c}{$\begin{array}{c}\delta t_{\mathrm{cal}} \\
(\mu \mathrm{s})\end{array}$} & $N$ & $N_{\mathrm{p}}$ & $N_{\mu}$ & $N_{\mu-\mathrm{QP}}$ & $N_{\text {no }-\mu}$ & $T$ & $S N R$ \\
\hline $0950+08$ & 0.253 & $2.9702^{a}$ & 89.1 & 14000 & 225 & 716 & 568 & 1 & 131072 & 0.29 \\
$1133+16$ (I) & 1.188 & $4.8471^{a}$ & 145.4 & 3000 & 240 & 362 & 544 & 41 & 524288 & 0.63 \\
$1133+16$ (II) & & & 145.4 & & 132 & 165 & 134 & 51 & 524288 & 0.33 \\
$1929+10$ & 0.226 & $3.1760^{b}$ & 95.3 & 15500 & $998(492)$ & 1093 & 602 & 14 & 262144 & 0.28 \\
\hline
\end{tabular}

${ }^{a}$ Phillips \& Wolszczan (1992).

${ }^{b}$ Manchester (1972).

for strong pulses. We used $S N R=0.1$ as a threshold for pulse selection. The approximate total number of pulses, $N$, observed and the number of selected pulses, $N_{\mathrm{p}}$, are listed for each pulsar in Table 1. This approach reduced the amount of data by a factor of several hundred and enabled us to carry out the subsequent signal processing more efficiently.

Having determined the pulse windows and selected strong pulses we further processed the recorded (raw) signal by decoding the signal amplitude sampled in two bits. Two-bit sampling of the amplitude of Gaussian random noise is widely used in VLBI observations. The decoding is generally done using four levels with integer values equal to $-3,-1,+1,+3$ (Thompson et al. 1988). These values reasonably represent the signal while the threshold level where the sampler switches from 1 to 3 and from -1 to -3 is equal to the current root-mean-square (rms) deviation ( $+1 \sigma$ and $-1 \sigma$, respectively) of the signal. In order to preserve this condition during observations, the S2 VLBI data acquisition system (S2-DAS) has an automatic gain control (AGC). For pulsar observations with the S2-DAS it is preferable to switch off the AGC and instead use the manual gain control with the gain fixed, or if left switched on, to choose a sufficiently long time constant for the AGC loop. Either option has the advantage of preventing the sampler from experiencing sudden gain discontinuities inside a pulse window. For our observations the AGC was inadvertently left switched on, but fortunately the gain was found to be constant inside the selected pulse windows in the majority of cases. Therefore the threshold level could relatively easily be adjusted during the analysis after the observations to reflect the larger voltage variations in the ON-pulse window. We changed the decoding values through the data records from \pm 1 and \pm 3 to real values that correspond to the current $\pm 1 \sigma$ levels in accordance with the technique developed by Jenet \& Anderson (1998). We computed the new levels from the quasi-instantaneous rms values of subsequent portions of the data records, each $100 \mu$ s long, to approximately match the dispersion smearing time across the 16- $\mathrm{MHz}$ bandwidth.
The next step in our data processing routine was the removal of the dispersion caused by the interstellar medium. The predetection dispersion removal technique itself (Hankins 1971) consists of a Fourier transform of the decoded signal followed first by amplitude corrections for the generally nonuniform receiver frequency bandpass and phase corrections for the dispersion delay, and then by an inverse Fourier transform back to the time domain. In particular, for the phase corrections of the dispersion delay, $\delta \Phi(v)$, at the observing frequency, $v$, we used

$\delta \Phi(v)=\delta \Phi\left(v_{0}+\Delta v\right)=\frac{2 \pi}{\alpha_{\mathrm{d}}} \cdot \frac{D M}{v_{0}^{3}} \Delta v^{2}\left(1-\frac{\Delta v}{v_{0}}\right)$,

where $\Delta v \ll v_{0}, D M$ is the dispersion measure, and $\alpha_{\mathrm{d}}$ is the dispersion constant (see caption of Table 1). Equation (1) was derived from Hankins \& Rickett (1975) by conserving only quadratic and cubic terms in their Eq. (5). The next term would give a correction of only about $10 \mathrm{~ns}$ for the dispersion smearing over the $16-\mathrm{MHz}$ bandwidth at $1650 \mathrm{MHz}$ for a typical value of $D M=3.0 \mathrm{pc} / \mathrm{cm}^{3}$ for our pulsars, which is only a sixth of our time resolution. Also, we did not need to apply a Doppler shift correction for DM due to the Earth's motion, since even for the largest possible velocity component of $30 \mathrm{~km} \mathrm{~s}^{-1}$ such a correction would be limited to a smearing across the $16-\mathrm{MHz}$ bandwidth of only about $40 \mathrm{~ns}$ for any of our pulsars which is smaller than our time resolution. The values of $D M$ used for the three pulsars are listed in Table 1. After the amplitude and phase corrections we set to zero all negative frequency components in the spectrum and then computed the inverse Fourier transform. The sum square of the real and imaginary components of the complex time series represents the dispersion-free detected signal of the pulsar (Born \& Wolf 1964).

Finally, we calibrated the intensities. Their scale is based on the system temperature which was recorded in a log file. Noise fluctuations of the unsmoothed detected signal of $1 \sigma$ in the OFF-pulse window correspond to $50 \mathrm{Jy}$. Dynamically changing the conversion threshold levels during the ON-pulse and OFF-pulse windows permitted us to avoid a possible bias in the decoding of the two-bit samples. 


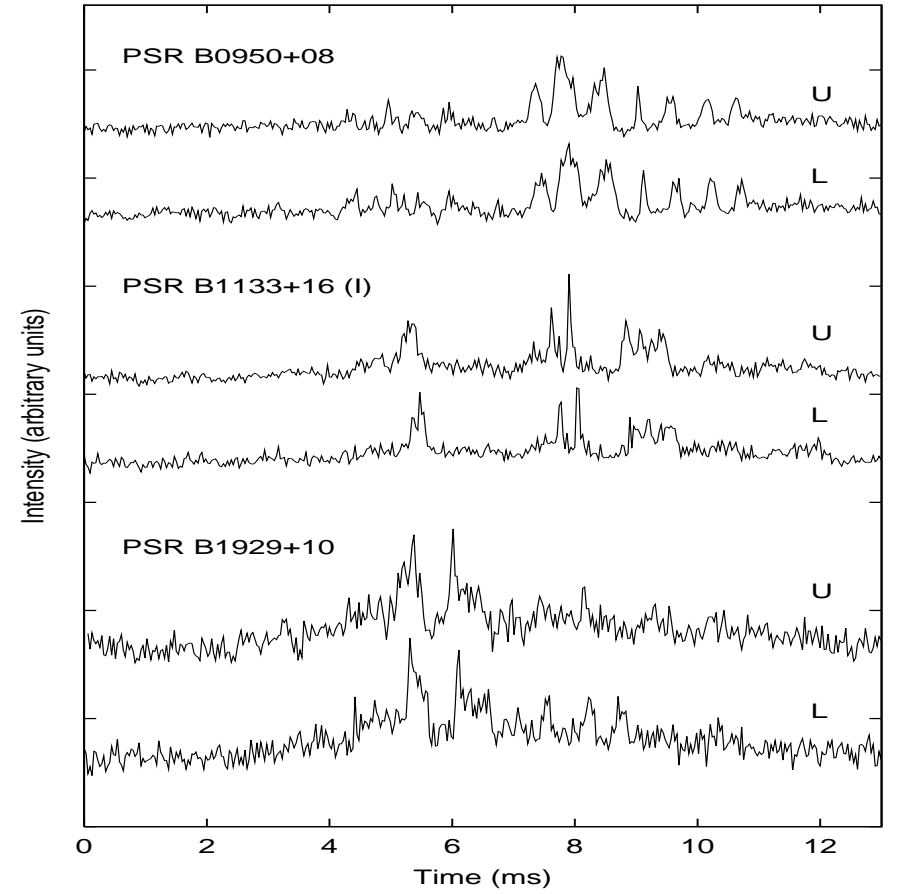

Fig. 1. An example of a strong pulse at $1650 \mathrm{MHz}(\mathrm{U})$ and $1634 \mathrm{MHz}$ (L) for each of the three pulsars. The dispersion smearing was removed, but the dispersion delay between frequency channels was not removed. The intensity fluctuations were smoothed over a 32- $\mu$ s time interval.

\section{Results}

\subsection{Individual pulses}

In Fig. 1 we display one example of a strong pulse for the upper (U) and lower (L) sideband for each of the three pulsars. The pulses are shown with a time resolution of $32 \mu \mathrm{s}$. It can be seen that the pulses vary in intensity on a timescale down to almost the resolution interval. The intensity variations are strongly correlated between the upper and lower frequency bands.

More rapid variations also appear to be present on first sight. For example, one very strong pulse of PSR B1133+16 has a mean flux density averaged over the pulse window of about $500 \mathrm{Jy}$, while the strongest isolated spike observed in the lower sideband has a flux density in excess of $6000 \mathrm{Jy}$. A short 4- $\mu$ s portion of this pulse is shown in Fig. 2 with the original time resolution of $62.5 \mathrm{~ns}$. For such short intensity variations in time no correlation is apparent between the data of the two sidebands even if allowance is made for a possible variation of $D M$ over a conceivable range of values. It is important to investigate whether these spikes and other strong unresolved ones are related to the physical emitters or are just statistically insignificant noise fluctuations of much broader pulse structure that increased the $\mathrm{ON}$-pulse antenna temperature and led to the strong variations.

In the remainder of the paper we first discuss the properties of the microstructure with a width of $\sim 1$ to $\sim 500 \mu \mathrm{s}$. Then we present a search for structure on sub-microsecond timescales,

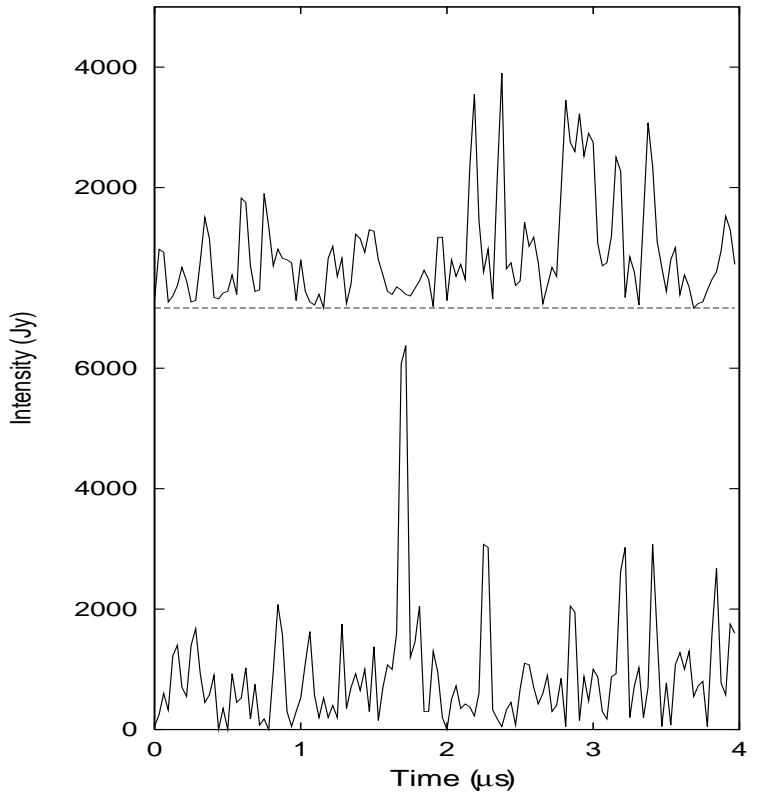

Fig. 2. A short portion of a very strong pulse from PSR B1133+16 plotted with a $62.5 \mathrm{~ns}$ time resolution. The upper curve corresponds to the $1650 \mathrm{MHz}$ frequency channel, and the lower one corresponds to the $1634 \mathrm{MHz}$ frequency channel. The dispersion smearing and time delay between the frequency channels were removed. The original 2bit quantization of the voltages is not visible because of the dispersion removal.

and finally we report on a study of the statistics of microstructure quasi-periodicities of the micropulses of the three pulsars.

\subsection{Microstructure}

\subsubsection{The typical microstructure width from the average cross-correlation function}

We determined the typical width of microstructure in a large number of single pulses from the width of the central spike of the averaged cross-correlation function (CCF). We chose CCFs for our analysis instead of the more widely used autocorrelation functions (ACFs) to avoid the noise spike at zero lag of the latter which could cause confusion in the detection of structure with the shortest timescales. More precisely, for each pulsar we selected $N_{\mathrm{p}}$ (Table 1) strong pulses, computed for each of them the CCF, $R_{1,2}(\tau)$, between the signals, $I_{1}(t)$ and $I_{2}(t)$, in the lower and upper sideband frequency channels, respectively, at lag $\tau$ with

$R_{1,2}(\tau)=\left[R_{1,1}(0) R_{2,2}(0)\right]^{-1 / 2} \sum_{t=1}^{T} I_{1}(t) I_{2}(t+\tau)$.

Here $t$ is the sample number at a particular time within the single pulse, $T$ (see Table 1) is the total number of samples chosen for the pulse window, and $I_{1}(t), I_{2}(t)$ represent ON-pulse intensities with the OFF-pulse mean levels subtracted. 
Table 2. Observed pulsar parameters: $\delta t_{\mathrm{obs}}$ is the observed time delay between the two frequency channels; $\delta t_{\mathrm{obs}}-\delta t_{\mathrm{cal}}$ is the difference between the observed and computed time delay for the two frequency channels; $\tau_{\mu \text {-broad }}$ and $\tau_{\mu \text {-narrow }}$ are the characteristic timescales of the microstructure determined from the average cross-correlation functions (see text); $\tau_{\mu \text {-shortest }}$ is the shortest width found for the observed micropulses; $t_{1 / 2}$ is the FWHM of the average profile or, in case of PSR B1133+16 (I), of the component in the profile (taken from Bartel et al. 1980). For PSR B1133+16 (II) no width is given in Bartel et al. (1980); the width is from Kramer (1994) interpolated between his values of a $F W H M$ of a Gaussian at 1.41 and $4.75 \mathrm{GHz}$ to $1.65 \mathrm{GHz}$. The parameter, $\tau_{\mathrm{m}}$, also from Bartel et al. (1980), is a measure of the typical subpulse width determined as the $50 \%$ width (ignoring the zero-lag spike) of the average ACF of single pulses (for PSR B1133+16 $\tau_{\mathrm{m}}=2.9 \pm 0.1 \mathrm{~ms}$, no distinction is made between the two components).

\begin{tabular}{lccccccc}
\hline \hline PSR B & $\begin{array}{c}\delta t_{\mathrm{obs}} \\
\mu \mathrm{s})\end{array}$ & $\begin{array}{c}\delta t_{\mathrm{obs}}-\delta t_{\text {cal }} \\
(\mu \mathrm{s})\end{array}$ & $\begin{array}{c}\tau_{\mu \text {-broad }} \\
(\mu \mathrm{s})\end{array}$ & $\begin{array}{c}\tau_{\mu \text {-narrow }} \\
(\mu \mathrm{s})\end{array}$ & $\begin{array}{c}\tau_{\mu \text {-shortest }} \\
(\mu \mathrm{s})\end{array}$ & $\begin{array}{c}t_{1 / 2} \\
(\mathrm{~ms})\end{array}$ & $\begin{array}{c}\tau_{\mathrm{m}} \\
(\mathrm{ms})\end{array}$ \\
\hline $0950+08$ & $87.2 \pm 0.3$ & $-1.9 \pm 0.3$ & $135 \pm 5$ & $14 \pm 3$ & 7 & 9.1 & 5.0 \\
$1133+16(\mathrm{I})$ & $143.1 \pm 0.2$ & $-2.3 \pm 0.2$ & $430 \pm 30$ & - & 6 & $6.0 \pm 0.6$ & - \\
$1133+16(\mathrm{II})$ & $143.1 \pm 0.2$ & $-2.3 \pm 0.2$ & $110 \pm 20$ & $11 \pm 3$ & 2 & $10.7 \pm 0.3$ & - \\
$1929+10$ & $95.2 \pm 0.1$ & $-0.1 \pm 0.1$ & $95 \pm 10$ & $9 \pm 3$ & 5 & $5.9 \pm 0.2$ & $3.4 \pm 0.1$ \\
\hline
\end{tabular}

We next averaged the CCFs for each pulsar and then corrected the averaged $\mathrm{CCF}$ for the receiver noise by dividing it by the factor $\epsilon$ (see Appendix A):

$\epsilon=1-\frac{\sigma_{\mathrm{off}_{1}}}{\sigma_{\mathrm{on}_{1}}} \frac{\sigma_{\mathrm{off}_{2}}}{\sigma_{\mathrm{on}_{2}}}\left(1+\frac{\left\langle I_{\mathrm{on}_{1}}\right\rangle}{\left\langle I_{\mathrm{off}_{1}}\right\rangle}+\frac{\left\langle I_{\mathrm{on}_{2}}\right\rangle}{\left\langle I_{\mathrm{off}_{2}}\right\rangle}\right)$.

The quantities $\left\langle I_{\mathrm{On}_{1}}\right\rangle,\left\langle I_{\mathrm{On}_{2}}\right\rangle$ represent the mean ON-pulse intensities inside the selected pulse window over $N_{\mathrm{p}}$ pulses for each of the bands, 1 and 2, after subtraction of the equivalent mean OFF-pulse intensities, $\left\langle I_{\mathrm{off}_{1}}\right\rangle,\left\langle I_{\mathrm{off}_{2}}\right\rangle$. The quantities, $\sigma_{\mathrm{on}_{1}}, \sigma_{\mathrm{on}_{2}}$, $\sigma_{\mathrm{off}_{1}}$ and $\sigma_{\mathrm{off}_{2}}$ are the rms deviations of $I_{\mathrm{on}_{1}}, I_{\mathrm{on}_{2}}, I_{\mathrm{off}_{1}}$, and $I_{\mathrm{off}_{2}}$, respectively, again averaged over $N_{\mathrm{p}}$ pulses. This expression corresponds to Eq. (20) in Rickett (1975).

Figure 3 shows the CCFs smoothed to a time-lag resolution of $1 \mu \mathrm{s}$ and shifted so that their time-lag origins correspond to the delays $\delta t_{\text {cal }}$, computed from the dispersion measure, $D M$ (see Table 1). The left column shows the CCFs on a relatively large time-lag scale while the right column displays only the central part of the CCFs. The maxima of the CCFs are close to the value of 0.5 predicted by the Amplitude-Modulated Noise (AMN) model (Rickett 1975). The relatively small discrepancies may be due to small and possibly insignificant errors of the correction factors for the CCFs.

For PSRs B0950+08 and B1929+10 a sharp spike can be clearly seen in the CCFs in the left column, indicating correlated microstructure in the two bands with a well defined characteristic timescale. For PSR B1133+16 a central spike is less pronounced in either of the two components at this frequency, but also existent.

We determined the characteristic timescale of the typical width of the microstructure from the intersection of the linear slope of the central narrow part (excluding the most inner section, see below) and the more slowly decreasing broader part of the CCFs by linear least-squares fits. We list the values with the corresponding statistical standard errors as $\tau_{\mu \text {-broad }}$ in Table 2.

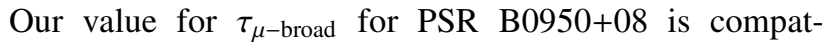
ible with earlier measurements between 130 and $200 \mu \mathrm{s}$ (Rickett 1975; Cordes \& Hankins 1977; Hankins \& Boriakoff 1978; Lange et al. 1998). For PSR B1133+16 microstructure was reported in the range of $\sim 340$ to $\sim 650 \mu$ s (Hankins 1972; Ferguson et al. 1976; Ferguson \& Seiradakis 1978;
Cordes 1976a; Popov et al. 1987; Lange et al. 1998), comparable to our value of component I but three to sixfold larger than that for component II. For PSR B1929+10 the width of microstructure has never been measured in the average ACF or CCF before. However, Lange et al. (1998) reported on the detection of structure on timescales around $150 \mu$ s close to their effective time resolution of $70 \mu \mathrm{s}$.

In the right column we display the central portions of the CCFs. For PSR B1133+16 (I) no additional abrupt sharpening of the CCFs can be seen. However for PSRs B0950+08, B1133+16 (II), and B1929+10 an additional central spike was found, indicating particularly short microstructure with characteristic timescales, $\tau_{\mu \text {-narrow }}$, of order $10 \mu$ s (see Table 2 ). Such short microstructure has never been seen in the average ACF or $\mathrm{CCF}$ of any pulsar. However, for the giant pulses from the Crab pulsar, microstructure was found in the ACF of a single pulse with the width at the $25 \%$ level of the ACF of $\leq 1 \mu$ s (Hankins 2000).

\subsubsection{Time delay of microstructure from the average cross-correlation function}

A close inspection of the inner part of the CCFs shows that the peak does not always occur exactly at the expected time delay. To quantify the discrepancy we measured the center of symmetry of the top part of the central portion of the CCFs in Fig. 3 and list it as $\delta t_{\text {obs }}$ in Table 2. To determine the standard errors, $\Delta \delta t_{\mathrm{obs}}$, we followed Chashei \& Shishov (1975) and used

$\Delta \delta t_{\mathrm{obs}} \sim \frac{4 \tau_{\mu}}{S N R}\left(N_{\mathrm{p}} T\right)^{-1 / 2}$,

where $\tau_{\mu}$ is the microstructure timescale, $\tau_{\mu \text {-broad }}$, listed in Table 2, and SNR is the signal-to-noise ratio of the intensity of the average single pulse integrated over the pulse window (Table 1).

For PSR B1929+10 the observed time delay is equal within $\Delta \delta t_{\text {obs }}$ to the expected time delay. However, for PSR B0950+08 and the first and second component of PSR B1133+16 there are significant discrepancies. The differences, $\delta t_{\mathrm{obs}}-\delta t_{\mathrm{cal}}$, are about $-2 \mu$ s (see Table 2) in the sense that micropulses at the higher frequency arrive slightly later than expected in comparison to 

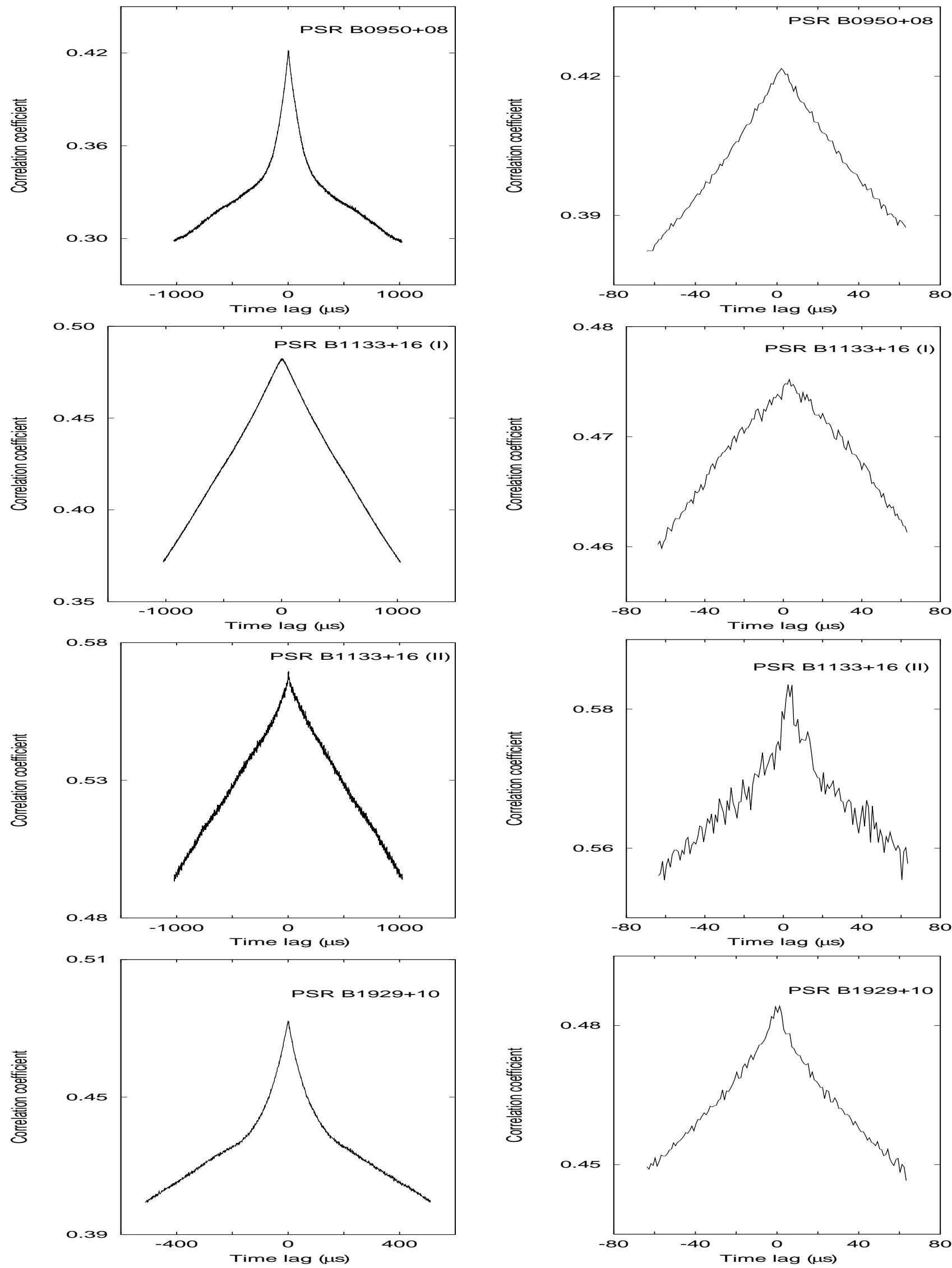

Fig. 3. The average cross-correlation function (CCF) for PSR B0950+08, the first (I) and second (II) component of PSR B1133+16, and PSR B1929+10. The CCFs were calculated for the unsmoothed ON-pulse intensities recorded in the conjugate 16-MHz bands and then smoothed over a time interval of $1 \mu \mathrm{s}$. The right column of the plots represents the very central portion of the CCFs shown in the left column. 

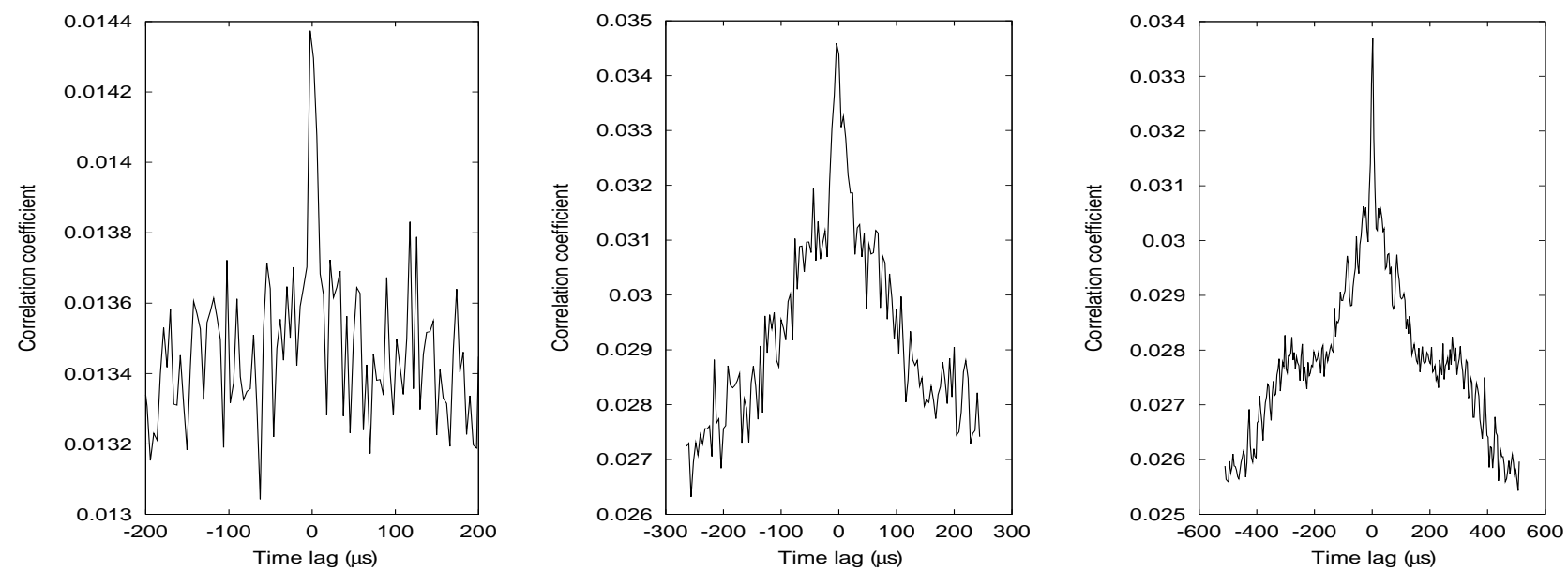

Fig. 4. Examples of individual CCFs for PSR B1133+16 (component II) in order of increasing complexity. The left plot shows microstructure with one notable width only. The middle and the right plots show microstructure with two and three widths. The scale of the correlation coefficients is not corrected for receiver noise.

the micropulses at the lower frequency. If interpreted as a dispersion measure difference, they correspond to values of $D M$, 1 to $2 \%$ smaller than those listed in Table 1 . However, as we will discuss in Sect. 6, we do not think that the values of $D M$ in Table 1 need to be corrected.

\subsubsection{The distribution of microstructure widths from individual cross-correlation functions}

In order to determine the distribution of microstructure widths for individual pulses, we computed the CCF for each of the $N_{\mathrm{p}}$ selected strong single pulses. Note that for PSR B1929+10 the number in Table 1 is given in parentheses, different from the number of pulses used to compute the average CCF. An interactive "TV"-based task was used to display the individual CCFs in a variety of timescales. With the help of a TV-cursor, the microstructure width was determined, again, from that point of the CCF where the central steep slope flattens out.

The majority of the selected strong pulses showed distinct short-term microstructure in the individual CCFs. Three examples of individual CCFs are shown in Fig. 4 for PSR B1133+16 (component II). The left plot shows a CCF with an individual spike only, the middle and the right plots show more complex CCFs with two and three different widths for the microstructure. For PSRs B0950+08 and B1929+10 very often several, up to five, different structural scales were found in the same pulse. In these cases several values for the microstructure width were obtained from one pulse. The total number of values for the microstructure width is listed for each pulsar (and each component in case of PSR B1133+16) in Table 1 as $N_{\mu}$. A number of single pulses had smooth CCFs without any distinct microstructure features. That number is given as $N_{\text {no- } \mu}$ in Table 1 . Only one from 225 pulses of PSR B0950+08, or less than $0.5 \%$ had no microstructure. For PSR B1929+10 about 3\% of single pulse had no microstructure, while for PSR B1133+16 about $17 \%$ and $38 \%$ of pulses had no microstructure in case of the first and the second component, respectively. This finding indicates even higher percentages of single pulses with microstructure than reported earlier (e.g., Smirnova et al. 1994;
Lange et al. 1998), if the results also hold for the weaker pulses which had to be ignored in our analysis because of sensitivity reasons.

The histograms of the microstructure widths are presented in Fig. 5. For all three pulsars they are skewed towards shorter widths. In general, they show a moderate rise from broader to shorter width starting at $\sim 200 \mu$ s for PSRs B0950+08 and $\mathrm{B} 1929+10$ and $\sim 800 \mu \mathrm{s}$ for the two components of PSR B1133+16. The rise becomes markedly sharper at $\sim 40 \mu \mathrm{s}$ for PSRs B0950+08 and B1929+10 and at $\sim 200 \mu$ s for each of the components of PSR B1 133+16, or at $\sim 0.08$ and $\sim 0.02 \%$ of $P$, respectively. The histograms peak at $\sim 20$ to $30 \mu$ s for PSRs B0950+08 and B1929+10 and $\leq 50 \mu$ s for the two components of PSR B1133+16. Towards shorter widths the histograms of PSRs B0950+08, B1133+16 (I, see inset of plot), and B1929+10 display a sharp cutoff at a width of $\sim 10 \mu \mathrm{s}$, and of PSR B $1133+16$ (II) at $<10 \mu$ s (not visible in plot). The shortest widths measured are $7 \mu \mathrm{s}$ for PSR B0950+08, $6 \mu \mathrm{s}$ and $2 \mu \mathrm{s}$ for PSR B1133+16 (component I and II, respectively), and $5 \mu \mathrm{s}$ for PSR B1929+10 (Table 2). The cutoff width and the shortest widths measured are greater than a) the smoothing interval of $1 \mu \mathrm{s}$ used in the analysis of the individual CCFs and b) the expected scattering time for either of the pulsars, and are therefore intrinsic properties of the pulsars. Again, there is a difference for the first and second component of PSR B1133+16. Micropulses with a width $\leq 50 \mu$ s occur almost twice as frequent in component II than in component I.

\subsection{Short-term noiselike intensity fluctuations}

Our average CCFs as well as our individual CCFs do not show any microstructure features with a sub-microsecond timescale. On the other hand, as was mentioned in Sect. 4.1, strong pulses when plotted with the highest time resolution contain bright sub-microsecond fluctuations (see Fig. 2). Also, Sallmen et al. (1999) reported to have found intensity fluctuations from the Crab pulsar which were still unresolved at their highest time resolution of $10 \mathrm{~ns}$. Is it possible that "nanopulses" exist in our data with a width not much larger than our highest time 

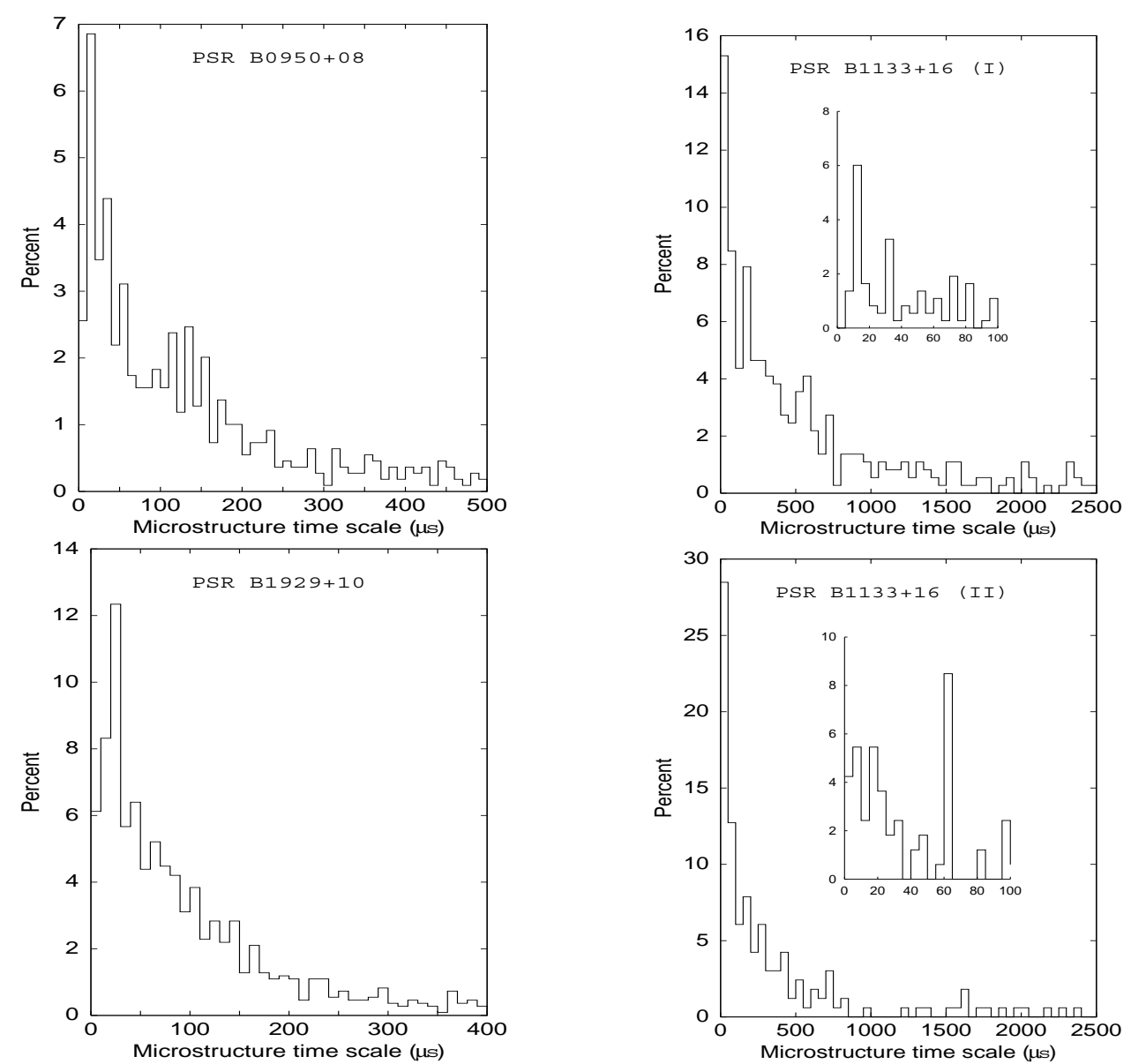

Fig. 5. Histograms of timescales of detected microstructure in the individual CCFs of single pulses for PSRs B0950+08, B1133+16 (components I, II), and B1929+10. The insets display histograms for the shortest timescales.

resolution of $62.5 \mathrm{~ns}$ ? They may perhaps not be visible in our histograms with more than 100 times wider bins. They could perhaps also be largely uncorrelated for the two bands and therefore not apparent in the CCFs. To investigate the significance of our fast intensity fluctuations we have to compare their statistics with the statistics of noise.

We use two approaches in our data analysis: 1) computation of short-term ACFs with a time-lag resolution of $62.5 \mathrm{~ns}$ for ON-pulse and OFF- pulse windows, and 2) comparison of the distribution of the intensities of short-term (62.5 ns) fluctuations ON-pulse with the distribution of such intensities OFFpulse and also with the $\chi^{2}$-distribution for thermal noise.

\subsubsection{Short-term ACFs}

Short-term ACFs, $R(\tau)$, with

$R(\tau)=\frac{1}{R(0)} \sum_{t=1}^{T}[I(t)-\langle I\rangle][I(t+\tau)-\langle I\rangle]$,

were computed for single pulses from many short adjacent intervals of the data with detected signal, $I(t)$, at sample number, $t$, both in ON-pulse and OFF-pulse windows. The short-time intervals were each $8 \mu$ s long and contained $T=256$ samples. The instantaneous mean level $\langle I\rangle$ used for the subtraction is given as $\langle I\rangle=\sum_{t=1}^{T} I(t) / T$. For each single pulse $m=256$
ACFs were computed for the ON-pulse window covering an interval of $2.048 \mathrm{~ms}$ centered close to the maximum of the selected pulses. The same number of ACFs was also computed for the OFF-pulse window of each single pulse. Note, that the definition of the ACF used here is not quite equivalent to the definition of the CCF in the Eq. (2).

In order to obtain sufficient sensitivity, we selected only very strong single pulses of number $k$ : 41 for PSR B0950+08, 24 for PSR B1133+16, and 30 for PSR B1929+10. For each of the pulsars no systematic difference was apparent between the ON-pulse and OFF-pulse average ACFs above the $3 \sigma_{\mathrm{ACF}}$ level. For an average ACF of white noise: $\sigma_{\mathrm{ACF}}=\frac{1}{\sqrt{T m k}}$, or $\sim 0.001$ in our cases. The corrections to the ON-pulse ACFs for receiver noise (see Eq. (3)) are only 5-10\%. Therefore, since no deviations were found between ON-pulse and OFF-pulse ACFs above the level of 0.003 , we conclude that submicrosecond micropulses, if present at all, do not contribute to the total power of pulsar signal variations by more than $0.3 \%$.

\subsubsection{Distribution of intensities}

We also compared the ON-pulse with the OFF-pulse intensity distributions and the $\chi^{2}$-distribution for thermal noise for the data with the highest resolution of $62.5 \mathrm{~ns}$. For each $8-\mu$ s interval with $T=256$ samples, the rms deviation $\sigma$ and the quantity, 
$(I(t)-\langle I\rangle) / \sigma$, were computed. The results obtained for three extremely strong pulses of PSR B1133+16 (component I) are presented in Fig. 6. Both distributions are fairly well fit by the theoretical curve for the $\chi^{2}$-distribution. The deviations at large amplitudes of both the ON-pulse and OFF-pulse distributions from the theoretical line can be contributed to effects of the two-bit sampling. In general, no notable differences are apparent between the ON-pulse and OFF-pulse distributions. ONpulse and OFF-pulse intensity fluctuations much shorter than $8 \mu$ s therefore have the statistics of thermal noise, consistent with the AMN model (Rickett 1975). The bright unresolved intensity spikes displayed in Fig. 2 are therefore insignificant statistical outbursts. No nanopulses were found.

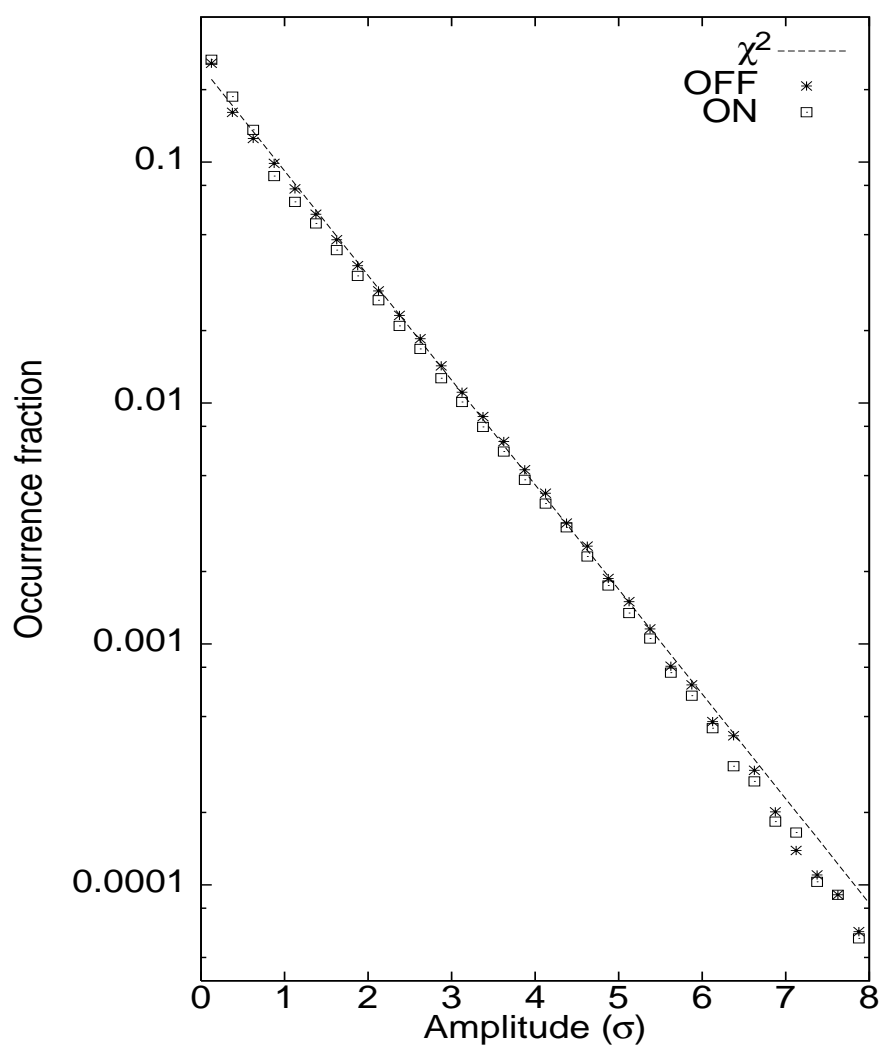

Fig. 6. Histograms of intensities at the highest resolution of $62.5 \mathrm{~ns}$ recorded ON-pulse (rectangles) and OFF-pulse (stars) for three extremely strong single pulses of PSR B1133+16 (component I). The dotted line corresponds to the $\chi^{2}$-distribution for thermal noise. The ordinate gives the number of occurrences as a fraction of the total number of intensity samples of 393216 . The abscissa gives the intensity in units of $\sigma$.

\section{Quasi-periodic microstructure}

\subsection{Analysis method}

Micropulse periodicities can be revealed, e.g., by analyzing ACFs, CCFs, or power spectra. Since our observations were conducted in two adjacent frequency bands, we again use CCFs and in addition cross-power spectra.
The cross-power spectrum is given as $\left|S_{1,2}\left(f_{\mu}\right)\right|^{2}$ with

$S_{1,2}\left(f_{\mu}\right)=\sum_{\tau=-T / 2}^{T / 2} R_{1,2}(\tau) W(\tau) \exp \left(-j 2 \pi f_{\mu} \tau\right)$,

where $f_{\mu}$ is the frequency of the microstructure periodicity, $R_{1,2}(\tau)$ is the CCF (see Eq. (2)), and $W(\tau)$ is Hanning's window (Blackman \& Tukey 1958) used to smooth variations of $S_{1,2}\left(f_{\mu}\right)$, with $W(\tau)=\frac{1}{2}\left[1+\cos \frac{2 \pi \tau}{T}\right]$.

Figure 7 shows an example of quasi-periodic microstructure within a single pulse of PSR B1133+16 (component I) for the upper and lower bands, the corresponding CCF, and its cross-power spectrum. The effect of the quasi-periodicity of the microstructure is clearly seen in the CCF and the cross-power spectrum.

In the following analysis we will use the cross-power spectra only. That has at least two advantages compared with the traditional use of ACFs and CCFs. First, in case of only one strong and isolated spectral line or feature as displayed in Fig. 7 the cross-power spectrum provides directly numerical values for the frequency, $f_{\mu}$, and width, $\Delta f_{\mu}$, of the detected feature that corresponds to the quasi-periodicity. Second, in case of more than one isolated strong feature, such features can be relatively easily distinguished in the cross-power spectrum but not so in an ACF or a CCF. We selected a feature as "detected" if its power spectral density was larger than, or equal to, $6 \mathrm{rms}$ in the spectrum on both neighboring sides of the feature, or if the power spectral density exceeded a reasonable threshold. The last condition was necessary to detect strong spectral features in the very low frequency range where the local rms variation could be overestimated because of the frequent complexity of the spectral features. In case of approximately symmetric features, the width, $\Delta f_{\mu}$, was determined as the $F W H M$. In case of complex features we interpreted the feature as a blend of several narrow features and estimated the FWHM of the dominant unblended feature by measuring the half-width at halfmaximum intensity from the apparently unblended side of the feature to the peak and then doubled that width.

In Table 1 we list the total number, $N_{\mu-\mathrm{QP}}$, of strong isolated features found in the cross-power spectra of $N_{\mathrm{p}}$ single pulses for PSRs B0950+08, B1133+16 (I, II), and B1929+10.

\subsubsection{Types of microstructure periodicities}

In Fig. 8 we show different types of cross-power spectra for several selected single pulses of PSR B1133+16. Similar types were also found for the other two pulsars. All spectra were normalized by their total power, i.e. the sum of all harmonics was set equal to unity.

Visual inspection shows that all spectral features below $10 \mathrm{kHz}$ are substantially broader than the applied frequency resolution of about $100 \mathrm{~Hz}$. The spectra therefore differ substantially from random noise and reflect the properties of the pulsar emission.

The spectra may be classified in four general categories in order of increasing complexity. The first category comprises spectra dominated by only one symmetrical feature (ae). The second category comprises spectra with two or more 

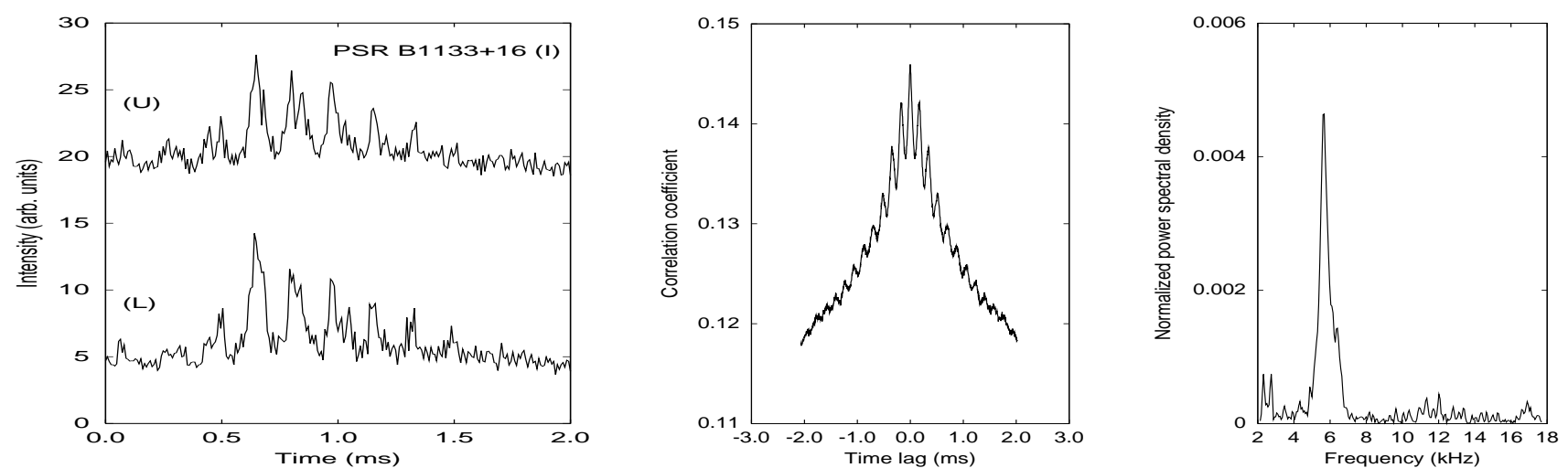

Fig. 7. An example of single pulse intensities with quasi-periodic microstructure for PSR B1133+16 (component I) recorded in the upper (U) and lower (L) sidebands (left) and the corresponding CCF (middle) and cross-power spectrum (right). For plotting purposes only, the intensities were smoothed with a time constant of $8 \mu \mathrm{s}$, and the CCF was smoothed with a time constant of $1 \mu \mathrm{s}$ and displayed with an arbitrary scale. For the cross-power spectrum we plot only the frequency range above $2 \mathrm{kHz}$, since the large power spectral density from features at lower frequencies would otherwise determine the scale completely.

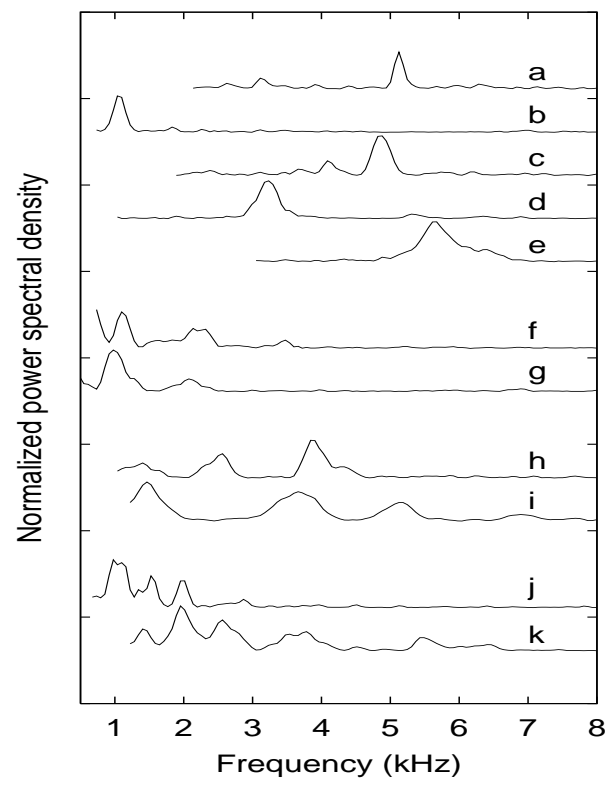

Fig. 8. Four different types of cross-power spectra (see text) for selected single pulses of PSR B1133+16 for any of its two components. Each spectrum is normalized by the total power, i.e. by the sum of the power of all harmonics. All plots shown in the figure start from some relatively high frequency. Low-frequency features are much more powerful and would determine the scale of the plots such that the high-frequency features would become almost invisible. The spectra were smoothed by using Hanning's window, reducing the originally high resolution only by $\sim 25 \%$. The noise level in the plots is much smaller than the features displayed. The spectrum (e) is a reproduction of the spectrum in the right panel of Fig. 7.

isolated symmetrical features with their frequencies being approximately multiple integers of the lowest frequency at which an isolated feature could be identified (f, g). These two categories correspond to the "classic" quasi-periodicities. However they constitute only about $5 \%$ of all analyzed spectra, about the same percentage for all three pulsars. The third category covers the spectra with several symmetrical isolated features located at more or less random frequencies (h, i). About $30-35 \%$ of all spectra fall under this category. Finally, the fourth category comprises spectra with isolated features at random frequencies as in the third category, but where each isolated feature is not symmetrical but instead structurally more complex $(\mathrm{j}, \mathrm{k})$. This is the most numerous category; it contains about two thirds of all spectra of all three pulsars.

\subsection{Histograms of the microstructure period}

In Fig. 9 we show the histograms of the microstructure period, $P_{\mu}\left(P_{\mu}=1 / f_{\mu}\right)$, for PSRs B0950+08, B1133+16 (I, II) and $\mathrm{B} 1929+10$. There are some general similarities between them. The histograms are skewed towards smaller microstructure periods. They have a relatively narrow peak at about $300 \mu$ s for PSRs B0950+08 and B1929+10 and a broader peak at $\sim 800 \mu \mathrm{s}$ for component I of PSR B1133+16 and $\sim 400 \mu$ s for component II, two to fourfold larger than $\tau_{\mu \text {-broad }}$. The histograms fall off sharply towards smaller periods. The widths of the histograms are relatively large. The bulk of microstructure periods (75\%) falls in the range of $0.1-1.0 \mathrm{~ms}$ for PSR B0950+08, 0.2-3.0 ms for component I of PSR B1133+16, 0.1-2.0 ms for component II of PSR B1133+16, and $0.2-0.7 \mathrm{~ms}$ for PSR B1929+10. For PSRs B0950+08 and B1133+16 for which microstructure results were published earlier, these range values agree well with those obtained by others at other frequencies with a smaller sample size (Hankins 1971; Soglasnov et al. 1981, 1983; Cordes et al. 1990; Smirnova et al. 1994).

Again, for PSR B1133+16 there is a clear difference in the histogram of microstructure periods. Quasi-periodicities with the shortest periods are relatively more numerous for the second component than for the first component.

\subsection{Histograms of the $Q$-factor of the microstructure periods}

The microstructure periods were determined from the peaks of the individual features in the cross-power spectra at frequency $f_{\mu}$. The widths, $\Delta f_{\mu}$, of the features varied widely. 

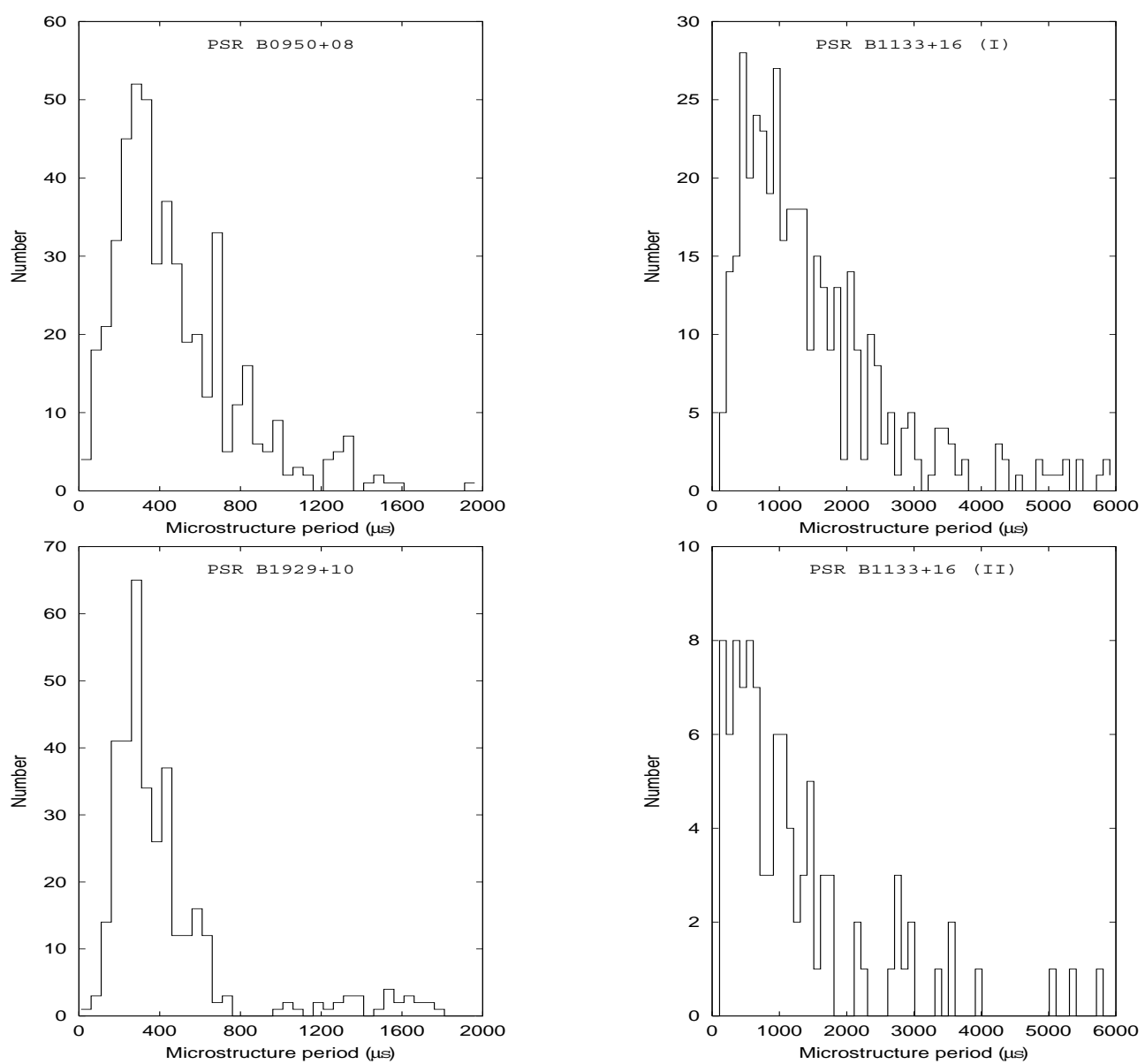

Fig. 9. Histograms of the microstructure periods, $P_{\mu}$, for the quasi-periodic features detected in the cross-power spectra.

To determine the sharpness of the features or the "fuzziness" of the microstructure periods we followed Cordes et al. (1990) and defined the $Q$-factor as $Q=f_{\mu} / \Delta f_{\mu}$. Figure 10 shows the histograms of the $Q$-factor for each of the three pulsars. A large majority of spectral features have relatively low values of $Q$ with the most frequent value of about 1.5 to 2 . In $75 \%$ of all cases $Q<5$ for PSR B0950+08 and each of the components of PSR B1133+16, and $Q<4$ for PSR B1929+10. However, some relatively high values with $Q \sim 10$ were also found, but only for PSR B0950+08 and the two components of PSR B1133+16.

\subsection{Average cross-power spectra}

In order to investigate the general aspects of the cross-power spectra we averaged the individual cross-power spectra for each of PSRs B0950+08 and B1929+10 and each component of PSR B1133+16. We then normalized each of the spectra so that they have equal power spectral densities at frequencies higher than about $50 \mathrm{kHz}$ where they are largely flat and identical to the equivalent spectra obtained for the OFF-pulse signal. In Fig. 11 we show these spectra for all three pulsars and compare them with the OFF-pulse spectra. At frequencies below about $20 \mathrm{kHz}$ the ON-pulse power spectra show a large excess above the noise power spectrum. This excess reflects the presence of microstructure fluctuation power with timescales down to $\sim 50 \mu \mathrm{s}$. The value corresponds to the timescales of microstructure revealed through the analysis of individual CCFs. The low frequency part can be approximated by the power law, $S \propto f^{-\alpha}$, with the exponent $\alpha \sim 2$ for all three pulsars. In comparison, the exponent for the OFF-pulse spectrum is about 0.5. Such an increase of power toward the low frequencies is typical for low-noise amplifiers. The noise with these characteristics is called excess noise, low-frequency noise, or $1 / f$ noise. Usually its exponent is in the range from 0.8 to 1.5 . Our rather low value of the exponent is probably due to the properties of the cross-power spectrum in our analysis. Clearly, the analysis of the average power spectrum is another way to detect the presence of microstructure and its quasi-periodicities.

\section{Discussion}

We have presented a statistical analysis of the properties of microstructure for PSR B0950+08, the two components of PSR B1133+16, and PSR B1929+10, the most extensive such analysis with one of the highest time resolutions (62.5 ns) ever made.

Microstructure with a characteristic broad timescale between $\sim 95$ and $\sim 450 \mu$ s in the ACF or CCF was confirmed for PSRs B0950+08 and B1133+16 and for the first time measured for PSR B1929+10. Microstructure with a characteristic short timescale of only $\sim 10 \mu$ s was found for PSR B0950+08, 

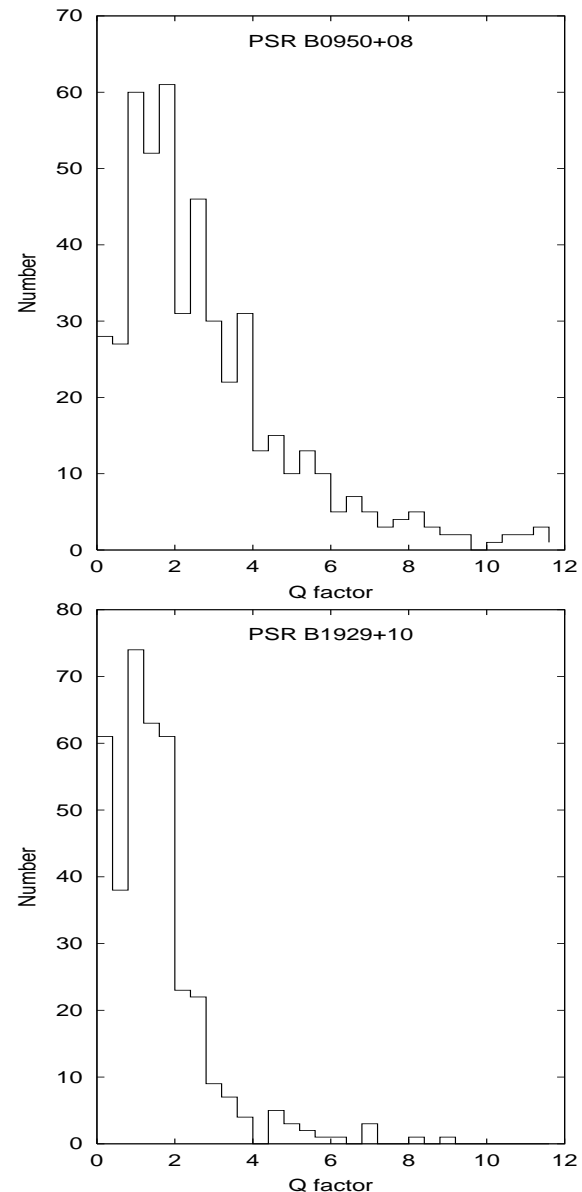

Fig. 10. Histograms of the microstructure period $Q$-factor.

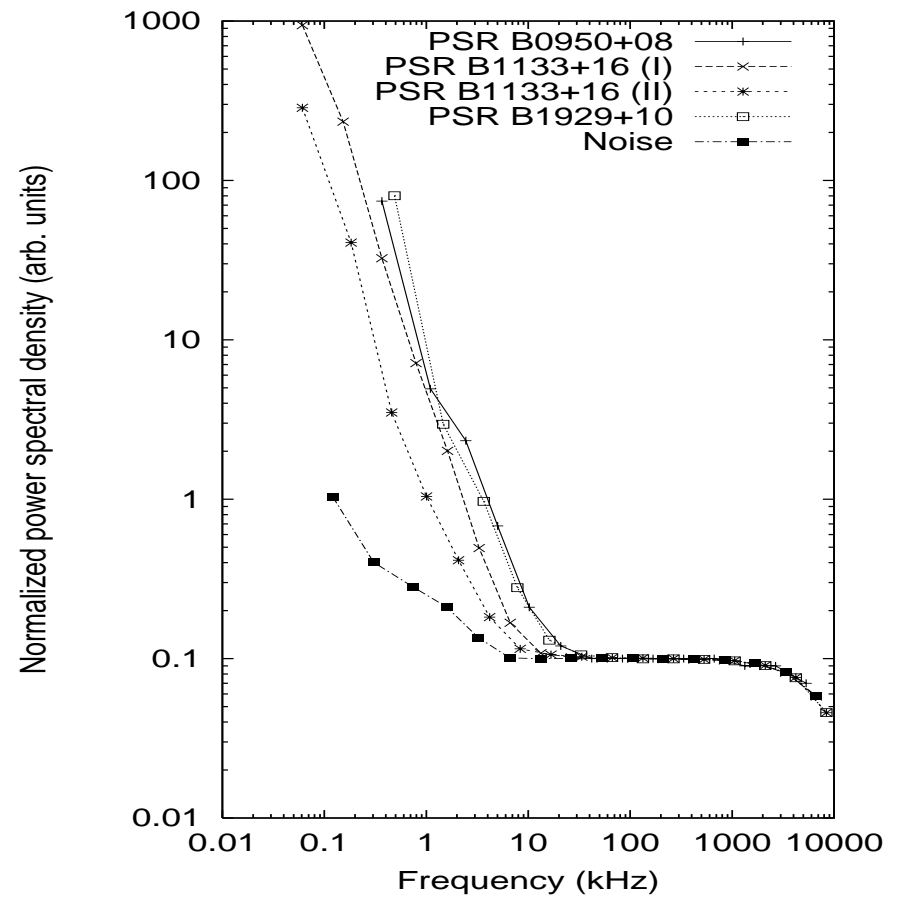

Fig. 11. Average cross-power spectra of the intensities at two conjugate 16-MHz bands for ON-pulse and OFF-pulse emission. The spectra were normalized to the largely flat part of the OFF-pulse spectrum between $50 \mathrm{kHz}$ and $1000 \mathrm{kHz}$.
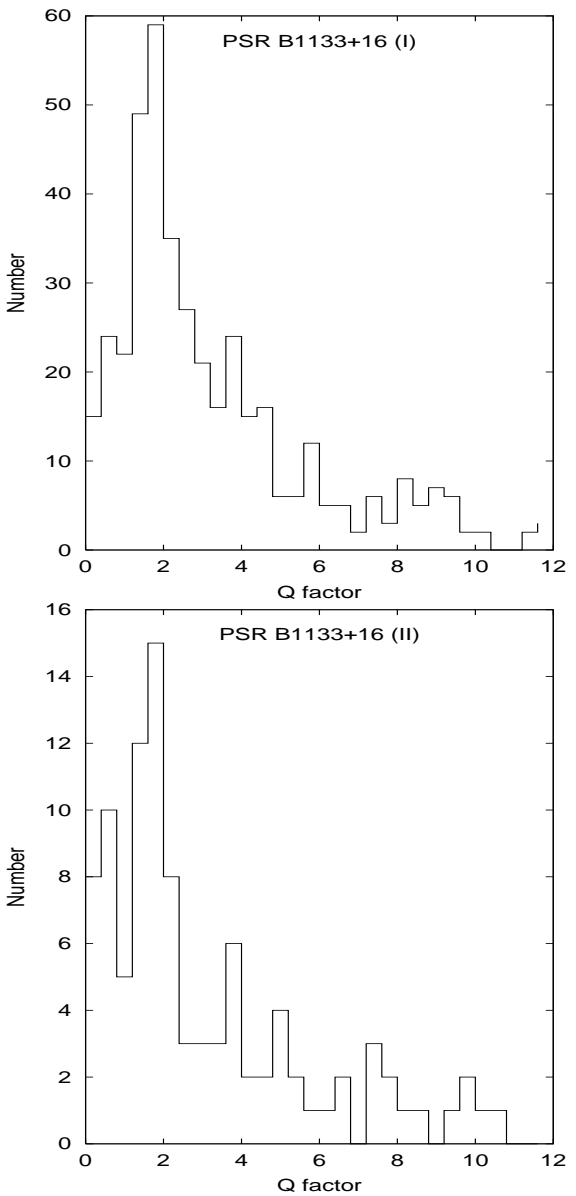

the second component of PSR B1133+16, and for PSR B1929+10, the first time such a short characteristic timescale was detected in the average ACF or CCF from any pulsar. Clearly, microstructure can have broad as well as short timescales for the same pulsar. This conclusion is further supported by the histograms of the microstructure timescales which generally showed a moderately rising part toward shorter timescales, corresponding to the broad micropulses, and a sharply rising part corresponding to short micropulses.

Different characteristic timescales for micropulses were previously found by others, albeit for much broader widths. Soglasnov et al. (1981) observed PSRs B0809+74 and $\mathrm{B} 1133+16$ at $102.5 \mathrm{MHz}$ with a time resolution of $10 \mu$ s and found that a long timescale of $\geq 500 \mu$ s and a shorter one of $\leq 500 \mu$ s are present in the ACFs of these pulsars. Later it was shown for the same pulsars that micropulses with a long timescale are clearly correlated over a broad frequency range of 70-102.5 MHz (Popov et al. 1987) while short timescale micropulses are not correlated over a narrow frequency range of 1.6 MHz at a center frequency of $102.5 \mathrm{MHz}$ (Smirnova et al. 1986). In contrast, in the present study we have found for PSRs B1133+16 (component II) and B1929+10 a clear correlation between the short timescale microstructure in the two adjacent bands separated by $16 \mathrm{MHz}$ or $1 \%$ of the center frequency.

Despite several hours of observations, no nanopulses were detected for any of the pulsars. The shortest micropulse was 
found for the second component of PSR B1133+16 and had a width of $2 \mu \mathrm{s}$. In particular, the histograms of the microstructure widths for all three pulsars showed a sharp cutoff at 5 to $10 \mu \mathrm{s}$. The rare occurrence of micropulses with short widths of only a few microseconds but with flux densities strong enough to be detected is consistent with earlier results for PSR B1133+16 at nearly the same frequency by Bartel (1978) and Bartel \& Hankins (1982) who also found only one or at best a few micropulses with such a short width after several hours of observations. Either micropulses with widths shorter than about $1 \mu$ s do not exist at our observing frequency in the three pulsars we studied or they are so weak or occur so infrequent that they have not yet been observed.

In contrast to our pulsars, Hankins (2000) reported to have found extremely strong fluctuations for the Crab pulsar which are still unresolved with a time resolution of $10 \mathrm{~ns}$. It remains to be seen whether the difference in the shortest timescale for microstructure for our three pulsars on the one hand and for the Crab pulsar on the other hand is caused by the Crab pulsar's young age, small period, or general uniqueness. Observations of other pulsars with a similarly high time resolution as ours are needed to investigate further this aspect of microstructure research.

Detailed inspection of the central portion of the average CCFs showed that the time delay of the micropulses between the two sidebands is for PSR B1929+10 within the error as expected, but is for PSRs B0950+08 and B1133+16 1 to $2 \%$ shorter than expected. The latter discrepancy has never been observed before. Earlier investigations of a possible discrepancy were based on observations with a coarser timing accuracy and a larger relative and absolute frequency difference of the two channels than ours (Boriakoff 1983; Popov et al. 1987; Sallmen et al. 1999).

The quasi-periodicities observed for all three pulsars were in most cases only relatively weakly pronounced with $Q$ factors generally between 1.5 and 2 and cross-power spectra showing rather complex features. Only in $5 \%$ of all cases did we detect classic quasi-periodicities with single spectral features and their harmonics. In fact, it appears that the complexity of the spectra almost continuously covers all of our four different categories. In this sense there is no fundamental difference between spectra of classic quasi-periodicities and spectra with no clear periodicities at all.

In this context the interpretation of microstructure quasiperiodicities in terms of modes of vibrations of neutron stars (Boriakoff 1976; van Horn 1980; Hansen \& Cioffi 1980) is not attractive although some modes fall well into the range of the observed quasi-periodicities (see McDermott et al. 1988). However, our values of the $Q$-factor are very small in comparison to about 1000 expected from stellar vibrations. Further, the essential continuum of the degree of complexity of the crosspower spectra and the lack of any resonance spike in the histograms for the microstructure period, $P_{\mu}$, make it very unlikely that the microstructure quasi-periodicities are caused by vibrations of the neutron star. Similar conclusions were drawn by Cordes et al. (1990) as a result of a study of five pulsars at several frequencies.
We find it most remarkable that the micropulses in the two components of PSR B1133+16 display different characteristics in every aspect we analyzed. The average CCFs, the histograms of microstructure timescales, the histograms of microstructure period, and the average cross-power spectra are all significantly different for the two components. Most striking is the more dominant occurrence of short micropulses in the second component which is reflected in the sharp spike of the central portion of its average CCF and the twofold higher frequency of occurrence in the histogram for $\leq 100 \mu$ s widths.

Differences in microstructure parameters for the two components of PSR B1133+16 were first reported by Cordes \& Hankins (1977) in their study of polarization properties of microstructure at $430 \mathrm{MHz}$ with a time resolution of $8 \mu \mathrm{s}$. Also, Smirnova et al. (1994) found that in the range from 200 to $1500 \mu \mathrm{s}$ micropulses of component II more frequently had smaller widths than those of component I in their observations at $1.4 \mathrm{GHz}$ with a time resolution of $150 \mu \mathrm{s}$. These results are at least qualitatively consistent with our result.

Are the micropulses we observe due to a longitudinal emission modulation and therefore to a sweep of the beam past the observer, or due to a radial emission modulation and therefore to the intrinsic time variability of the emission, or perhaps due to a combination of both? The average pulse profile clearly reflects the beam pattern; the equivalent profile width is proportional to $P$ (Lyne \& Manchester 1988). Subpulses in single pulses have a typical width $\propto P^{0.8}$ indicating that the millisecond structure like the average pulse profile can largely be interpreted as being due to the sweep of the beam past the observer (Bartel et al. 1980). Broad micropulses of several $100 \mu \mathrm{s}$ duration were reported to have a somewhat weaker dependence on period ( $\propto P^{0.5}$; Taylor et al. 1975; see also Ferguson 1977) reflecting perhaps already part of the elementary emission mechanism with its temporal variations.

Three pulsars are not enough to test this relation since the scatter about the regression line is relatively large. However, we can compare our broad, narrow, and shortest microstructure widths with the component widths of the average profile, $t_{1 / 2}$, and with the subpulse widths, $\tau_{\mathrm{m}}$, listed for the three pulsars in Table 2. The ratios for the comparison are given in Table 3. While the microstructure widths relative to $P$ can indeed vary within their categories by several times their errors, they are constant within about twice the combined errors relative to $t_{1 / 2}$ and $\tau_{\mathrm{m}}$ (with the exception of $\tau_{\mu \text {-broad }}$ relative to $t_{1 / 2}$ for component I of PSR B1133+16).

In other words, with the exception of the broad micropulse widths for PSR B1133+16 (I), each of the different types of micropulse width scales within $\sim 2 \sigma$ with the subpulse width and the average pulse component width. That may be an intriguing result. It therefore appears possible that even the fastest observable intensity fluctuations still have a strong component of the longitudinal emission modulation and are at least partly due to the sweep of the beam. If so, we can compute the factor $\gamma$ as the energy relative to the rest energy of the particles moving along 
Table 3. Comparison of pulsar parameters and $\gamma$ factors. The parameters for comparison in Cols. $2-4$ are taken from Table 2. For PSR B0950+08 $3 \%$ errors are assumed for $t_{1 / 2}$ and $\tau_{\mathrm{m}}$. The parameter $\gamma_{\mu \text {-narrow }}^{\left(\zeta=90^{\circ}\right)}$ is derived for an angle, $\zeta$, between the line of sight and the rotation axis of the pulsar of $90^{\circ}$ and $\gamma_{\mu \text {-narrow }}$ for an angle estimated from the polarization angle sweep across the beam for the hollow-cone model, $\zeta=10.1,55.0$, 10.0 for PSRs B0950+08, B1133+16 (II), and B1929+10, respectively (Lyne \& Manchester 1988). For the first two pulsars the values for $\zeta$ are similar to those given by Rankin (1993). For PSR B1929+10 Rankin (1993) interpreted the viewing geometry very differently in comparison to Lyne \& Manchester (1988), but did not give a value of $\zeta$. The errors in Cols. 5-6 are "symmetrized" errors based on the errors of $\tau_{\mu-\text { narrow }}$ only.

\begin{tabular}{|c|c|c|c|c|c|c|}
\hline PSR B & $\begin{array}{c}\tau_{\mu-\text { broad }} \\
\left(10^{-4} P\right) \\
\left(10^{-2} t_{1 / 2}\right) \\
\left(10^{-2} \tau_{\mathrm{m}}\right)\end{array}$ & $\begin{array}{c}\tau_{\mu \text {-narrow }} \\
\left(10^{-5} P\right) \\
\left(10^{-3} t_{1 / 2}\right) \\
\left(10^{-3} \tau_{\mathrm{m}}\right)\end{array}$ & $\begin{array}{c}\tau_{\mu \text {-shortest }} \\
\left(10^{-3} \tau_{\mathrm{m}}\right)\end{array}$ & $\gamma_{\mu-\text { narrow }}^{\left(\zeta=90^{\circ}\right)}$ & $\gamma_{\mu-\text { narrow }}$ & $\begin{array}{c}c \cdot \tau_{\mu-\text { narrow }} \\
(\mathrm{km})\end{array}$ \\
\hline $0950+08$ & $\begin{array}{l}5.3 \pm 0.2 \\
1.5 \pm 0.1 \\
2.7 \pm 0.1\end{array}$ & $\begin{array}{l}5.5 \pm 1.2 \\
1.5 \pm 0.3 \\
2.8 \pm 0.6\end{array}$ & $1.40 \pm 0.04$ & $2900 \pm 700$ & $16400 \pm 4000$ & $4.2 \pm 0.9$ \\
\hline $1133+16(\mathrm{I})$ & $\begin{array}{c}3.6 \pm 0.3 \\
7.2 \pm 0.9 \\
-\end{array}$ & $\begin{array}{l}- \\
- \\
-\end{array}$ & - & - & - & - \\
\hline $1133+16$ (II) & $\begin{array}{c}0.9 \pm 0.2 \\
1.0 \pm 0.2 \\
-\end{array}$ & $\begin{array}{c}0.9 \pm 0.3 \\
1.0 \pm 0.3 \\
-\end{array}$ & - & $16200 \pm 5000$ & $19700 \pm 6000$ & $3.3 \pm 0.9$ \\
\hline $1929+10$ & $\begin{array}{l}4.2 \pm 0.4 \\
1.6 \pm 0.2 \\
2.8 \pm 0.3\end{array}$ & $\begin{array}{l}4.0 \pm 1.3 \\
1.5 \pm 0.5 \\
2.7 \pm 0.9\end{array}$ & $1.47 \pm 0.04$ & $4000 \pm 1500$ & $23000 \pm 9000$ & $2.7 \pm 0.9$ \\
\hline
\end{tabular}

the curved magnetic field lines and beaming in the direction of motion. For point sources,

$\gamma=\frac{1}{\phi \cdot \sin \zeta}=\frac{P}{2 \pi \sin \zeta \cdot \tau_{\mu-\text { narrow }}}$

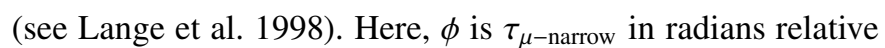
to $P$, and $\zeta$ is the angle between the rotation axis of the pulsar and the line of sight to the observer. For the conservative assumption of $\zeta=90^{\circ}$ the values of $\gamma$ differ by up to a factor of 5. However, if the angle $\zeta$ is taken from estimates obtained from the sweep of the polarization angle across the pulse profile (Lyne \& Manchester 1988), the values for $\gamma$ are much more uniform with a mean of about 20000 and a spread of not more than a factor 1.4 which is well within the errors. Perhaps such a high value for $\gamma$ is representative for the relativistic energy of the particles.

Any longitudinal emission pattern of the beam on this small angular scale is likely related to the radial pattern through the radius to frequency relation and the curved open magnetic field lines of the polar regions. For instance, for PSR B0950+08 the subpulse ACF width increases from $4.2 \mathrm{~ms}$ at $2.7 \mathrm{GHz}$ to $5.0 \mathrm{~ms}$ at $1.7 \mathrm{GHz}$ (Bartel et al. 1980), reflecting the above frequency dependent geometry. Since the subpulses can be approximated to first order as Gaussians ${ }^{1}$, the $50 \%$ ACF width has to be scaled upwards by a factor of 1.4 to yield the FWHM of the subpulses. If we had observed the subpulses over a continuous bandwidth from 2.7 to $1.7 \mathrm{GHz}$, they would be smeared in

\footnotetext{
${ }^{1}$ A detailed analysis showed that the average ACF of subpulses of a representative sample of pulsars, and by implication the average subpulse itself, is sharper at the top than either a Gaussian or a Lorenzian but flatter than a Gaussian and similar to a Lorenzian in the wings (Bartel et al. 1980).
}

time at the wings by about a third of the difference of the scaled widths or $0.37 \mathrm{~ms}$. On average, the smearing time for a subpulse would be about $0.19 \mathrm{~ms}$. Correspondingly, we could expect our micropulses observed over a total of the two bands of $32 \mathrm{MHz}$ to be smeared in time on average by about $6.1 \mu \mathrm{s}$. For PSRs B1133+16 and B1929+10 the expected smearing times based on the differences of the scaled ACF widths are 4.5 (see caption of Table 2 for PSR B1133+16) and $3.7 \mu$ s, respectively. The expected smearing times for the first and third pulsar are in good agreement with their shortest widths. For PSR B1133+16 the ACF width refers to a weighted mean of the ACF widths of both components. The mean of our shortest widths for both components is $4.5 \mu \mathrm{s}$ which equally well agrees with the expected smearing time for both components.

In this context the residual delay of $\sim 2 \mu$ s that we observed for the microstructure of the two bands for two pulsars can be interpreted as being due to the curved geometry of the magnetic field lines and to the radius to frequency mapping. One might expect the delay to be a function of the phase of the micropulses in the pulse window. Micropulses in the leading part of the pulse window would first appear at the lower frequency, micropulses in the trailing part first at the higher frequency. In case of PSR B1133+16 for which we distinguished between a leading and a trailing component we did indeed find that the lower frequency micropulses arrived first in the leading component. However, we did not observe a sign reversal of the delay for the micropulses in the second component.

This complication in the interpretation notwithstanding, it is conceivable that the smallest observable angular scale of the fluctuating radiation pattern of the beam is limited by the geometry of the opening cone of emission and therefore likely by the opening polar magnetic field lines, the particular radius to 
frequency mapping, and the bandwidth of the receiver. Shorter pulses in these pulsars may become observable by using a smaller bandwidth. The shortest pulses observable at $1.7 \mathrm{GHz}$ would be limited by the rise time of the optimal filter to about $0.5 \mu \mathrm{s}$. Other pulsars may have a more favorable, weaker dependence of the ACF width on frequency and could show less angular smearing. A detailed study of the correlation and the pulse time of arrivals of micropulses at several close frequencies with a similar resolution of 62.5 ns could provide important clues about the geometry of the magnetic field lines, the elementary pulse structure, and in turn the emission mechanism.

However, the alternate interpretation of the short micropulses being due to a radial modulation of the radiation pattern is not necessarily disfavored. In the context of this interpretation the values for $\gamma$ could be easily two to three orders of magnitude lower and the typical radial length of the modulation $\sim c \cdot \tau_{\mu \text {-narrow }}$ (see Table 3 ). The sources of such short micropulses have brightness temperatures of $\sim 10^{34} \mathrm{~K}$. With the influence of beaming being excluded, the high degree of coherence of the modulation over the radial length would primarily account for the high brightness temperature.

Several possible emission mechanisms have been proposed to explain radio emission of pulsars. In general, they can be classified into three groups: emission by bunches, plasma instabilities, and maser emission (Melrose 1992). Theoretical predictions on the possible generation of ultra short timescale intensity fluctuations in the pulsar radio emission are based on nonlinear temporal models describing the interaction of a high energy beam of particles with plasma wave packets in the pulsar magnetosphere. Asseo et al. (1990) suggested that a twostream instability may result in the growth of strong plasma turbulences which would lead to a self-modulation instability, the creation of localized wave packets, and the generation of stable Langmuir soliton-like solutions. The latter would result in the so-called "Langmuir microstructures" with timescales in the range of several microseconds. Our shortest micropulses have the predicted typical durations and could be interpreted in this way.

Other interpretations of microstructure are based on nonlinear models of self-modulational instabilities of an electromagnetic wave with a large amplitude propagating in an electronpositron plasma (Chian \& Kennel 1983; Onishchenko 1990; Chian 1992; Gangadhara et al. 1993). The models demonstrate that the modulation instability can evolve to a nonlinear stationary state that results from the balance between nonlinearity and dispersion. The possible stationary solutions of the respective nonlinear equations are envelope solitons or isolated wave packets and periodic wave trains. The first solution may describe strong isolated micropulses while the second could describe the phenomenon of quasi-periodicity.

Recently Weatherall (1998) conducted a numerical simulation based on nonlinear wave dynamics which provides detailed solutions for the temporal behavior of pulsar radio emission. The model predicts an intrinsic pulse width in the range of 1-10 ns (nanostructure). The characteristic timescale is expected to be frequency dependent, becoming longer at lower frequencies.
Our analysis of pulsar radio emission with the resolution of $62.5 \mathrm{~ns}$ has not detected any nanopulses. Nanostructure may in general be present in radio emission as "shot noise" as suggested by Cordes (1976b). Perhaps nanopulses might be observed as an infrequent phenomenon like the "giant" pulses from the Crab pulsar.

However, no matter how short elementary pulses could intrinsically be, they are not only likely affected by angular smearing as suggested for our pulsars above but could perhaps also be significantly affected while propagating through the magnetosphere toward the observer. Lyutikov \& Parikh (2000) have studied the scattering and diffraction of radio waves in the pulsar magnetosphere and found that the scattering delay can be as large as $10 \mu \mathrm{s}$, easily explaining our cutoff widths in the histograms. The dispersion delay of micropulses of about $2 \mu \mathrm{s}$ we found for PSR B0950+08 and both components of PSR B1133+16 could also be explained in their model as a propagation effect.

The difference in the characteristics of microstructure for the two components of PSR B1133+16 remains puzzling. If the two components define the longitudes where the hollow cone of emission is intersected by the line of sight, then the emission mechanism produces micropulses with characteristics as a function of azimuth about the axis of the hollow cone. Perhaps asymmetries in the polar cap magnetic field structure could cause azimuthally dependent plasma bunching properties and propagation effects that would result in the differences we observe.

\section{Conclusions}

The main results and the conclusions of our study can be summarized as follows:

1. The S2 VLBI system provided a conceptually clearly defined continuous flow of data with a time resolution of $62.5 \mathrm{~ns}$ that could be transferred via the S2-TCI to small digital tapes for the analysis of pulsar radio signals.

2. Broad microstructure was found for the first time in the average CCF (or ACF) of PSR B1929+10 and confirmed for PSRs B0950+08 and B1133+16.

3. Narrow microstructure with a typical width of only about $10 \mu \mathrm{s}$ was discovered in the average CCF of PSRs B0950+08, B1133+16 (component II) and B1929+10.

4. Histograms of microstructure timescales show a steep increase toward shorter timescales followed by a sharp cutoff at about 5-10 $\mu \mathrm{s}$. The shortest micropulse detected had a width of $2 \mu \mathrm{s}$.

5. No unresolved nanopulses or pulse structure with a submicrosecond timescale were found. This conclusion follows from the comparison of histograms of amplitudes for ON-pulse and OFF-pulse windows; it is confirmed by the completely identical shapes of the high-frequency portions of the average cross-power spectra for ON-pulse and OFFpulse emission; and it is supported by the short-term ACFs which show that the pulsar signal is indistinguishable from pure receiver noise at timescales shorter than $8 \mu \mathrm{s}$. 
6. For PSR B1929+10 the dispersion delay for micropulses over $16 \mathrm{MHz}$ or $\sim 1 \%$ of the center frequency is the same within one standard error as that for the average pulse over a much larger absolute and relative frequency spread. For PSRs B0950+08 and B1133+16 the residual delay is about $2 \mu$ s with the micropulses at the lower frequency arriving earlier than expected.

7. The cross-power spectra for single pulses display features with an almost continuous variety of complexity ranging from single features that correspond to the classic quasiperiodicities to multiple and broad overlapping features with random positions in the spectra that correspond to essentially no quasi-periodicities at all.

8. The power spectral density of the average cross-power spectra of single pulses decreases steeply toward higher frequencies of the microstructure quasi-periodicities and merges with the power spectral density of the OFF-pulse emission at a frequency that corresponds to the shortest microstructure quasi-periodicities.

9. The statistics of the micropulses and their quasiperiodicities differ significantly for the two components of PSR B1133+16.

10. It is conceivable from our data that the shortest micropulses represent, at least in part, the shortest angular scale of the longitudinal radiation pattern of the sweeping beam with $\gamma \sim 20000$ for the radiating particles. The particular combination of the geometry of the opening, curved magnetic filed lines, the radius to frequency mapping, and the bandwidth over which the radiation is observed limited the observable width of micropulses in our observations to $\sim 5 \mu \mathrm{s}$. The shortest micropulses of our three pulsars at $1.7 \mathrm{GHz}$ are predicted to have a width of $\sim 0.5 \mu \mathrm{s}$. If the short micropulses are instead due to a radial modulation of the radiation pattern the typical length of the pattern is $\sim 3 \mathrm{~km}$.

Acknowledgements. We thank the anonymous referee for very helpful suggestions for an improvement of the paper. M. V. Popov thanks the Space Geodynamics Laboratory at CRESTech and York University for providing support for his work on the data reduction while he was staying in Toronto. N. Bartel thanks the Astro Space Center of the Lebedev Physical Institute in Moscow, the Canadian Institute for Theoretical Astrophysics (CITA) in Toronto, and the Observatório Nacional in Rio de Janeiro for their hospitality and support during part of his sabbatical year while this paper was being written. This investigation was supported in part by the Russian Foundation for Fundamental Research (project's Nos. 98-02-16917 and 01-0216871), by INTAS (project No. 96-0154), and by Canada's NSERC. The DSN 70-m telescope is operated by JPL/Caltech under contract with the National Aeronautics and Space Administration.

\section{Appendix A: Correction of the average CCF for the effects of receiver noise}

The effects of receiver noise on the average ACF of a pulsar signal were investigated by Rickett (1975). The ACF, $r(\tau)$, of the pulse intensity in terms of observable quantities is given as

$$
\langle r(\tau)\rangle=\left\langle r_{\text {on }}(\tau)\right\rangle-\left\langle r_{\text {off }}(\tau)\right\rangle\left(1+2 \frac{\left\langle I_{\text {on }}\right\rangle}{\left\langle I_{\text {off }}\right\rangle}\right),
$$

where $r_{\mathrm{on}}(\tau)$ and $r_{\mathrm{off}}(\tau)$ are the ACFs for the ON-pulse and OFF-pulse windows each averaged over all pulses. Further, $\left\langle I_{\mathrm{on}}\right\rangle$ is the mean intensity in the respective window again averaged over all pulses, but with $\left\langle I_{\text {off }}\right\rangle$ subtracted. The normalized $\mathrm{ACF}, R(\tau)$, is then given as

$\langle R(\tau)\rangle=\frac{\langle r(\tau)\rangle}{\langle r(0)\rangle}=\frac{\left\langle r_{\text {on }}(\tau)\right\rangle}{\left\langle r_{\text {on }}(0)\right\rangle-\left\langle r_{\text {off }}(0)\right\rangle\left(1+2 \frac{\left\langle I_{\text {on }}\right\rangle}{\left\langle I_{\text {off }}\right\rangle}\right)}$,

$\left\langle r_{\text {off }}(\tau)\right\rangle$ being essential zero for $\tau>1 / B$, where $B$ is the receiver bandwidth.

This equation can be rewritten in terms of $\left\langle R_{\mathrm{on}}(\tau)\right\rangle=\frac{\left\langle r_{\mathrm{on}}(\tau)\right\rangle}{\left\langle r_{\mathrm{on}}(0)\right\rangle}$ as:

$\langle R(\tau)\rangle=\frac{\left\langle R_{\mathrm{on}}(\tau)\right\rangle}{\epsilon}$,

where $\epsilon$ is a correction factor:

$\epsilon=1-\frac{\sigma_{\mathrm{off}}^{2}}{\sigma_{\mathrm{on}}^{2}}\left(1+2 \frac{\left\langle I_{\mathrm{on}}\right\rangle}{\left\langle I_{\mathrm{off}}\right\rangle}\right)$

with $\sigma_{\text {on }}=\sqrt{\left\langle r_{\text {on }}(0)\right\rangle}$ and $\sigma_{\text {off }}=\sqrt{\left\langle r_{\text {off }}(0)\right\rangle}$.

In this paper we used the $\mathrm{CCF}$ between adjacent $16-\mathrm{MHz}$ frequency channels. It can be seen that for the CCF the correction factor $\epsilon$ should be modified to:

$\epsilon=1-\frac{\sigma_{\mathrm{off}_{1}}}{\sigma_{\mathrm{on}_{1}}} \frac{\sigma_{\mathrm{off}_{2}}}{\sigma_{\mathrm{on}_{2}}}\left(1+\frac{\left\langle I_{\mathrm{On}_{1}}\right\rangle}{\left\langle I_{\mathrm{off}_{1}}\right\rangle}+\frac{\left\langle I_{\mathrm{on}_{2}}\right\rangle}{\left\langle I_{\mathrm{off}_{2}}\right\rangle}\right)$.

In fact, in our observations, the values of $\sigma_{\text {on }}, \sigma_{\text {off }},\left\langle I_{\text {on }}\right\rangle$, and $\left\langle I_{\text {off }}\right\rangle$ in one channel never differed from the equivalent ones by more than a few percent in the other channel because of the proximity of the two frequency channels and the AGC operation.

\section{References}

Arons, J. 1983, ApJ, 266, 215

Asseo, E., Pelletier, G., \& Sol, H. 1990, MNRAS, 247, 529

Backer, D. C. 1973, ApJ, 182, 245

Bartel, N. 1978, A\&A, 62, 393

Bartel, N., \& Hankins, T. H. 1982, ApJ, 254, L35

Bartel, N., Sieber, W., \& Graham, D. A. 1980, A\&A, 87, 282

Benford, G. 1977, MNRAS, 179, 311

Blackman, R. B., \& Tukey, J. W. 1958, Bell System Tech. J., 37, 185

Boriakoff, V. 1976, ApJ, 208, L43

Boriakoff, V. 1983, ApJ, 272, 687

Born, M., \& Wolf, E. 1964, Principles of Optics (Pergamon Press)

Cannon, W. H., Baer, D., Feil, G., et al. 1997, Vistas Astron., 41, 297

Chashei, I. V., \& Shishov, V. I. 1975, SvA Lett., 1, 9, transl. from: Pis'ma v AZh, 1975, 1, 18

Chian, A. C. L. 1992, in Proc. of IAU Colloq. 128, The Magnetospheric Structure and Emission Mechanisms of Radio Pulsars, ed. T. H. Hankins, J. M. Rankin, \& J. A. Gil (Zielona Gora: Pedagogical Univ. Press), 356

Chian, A. C. L., \& Kennel, C. F. 1983, Ap\&SS, 97, 9

Cordes, J. M. 1976a, ApJ, 208, 944

Cordes, J. M. 1976b, ApJ, 210, 780

Cordes, J. M. 1981, in Proc. of IAU Symp. 95, Pulsars: 13 years of research on neutron stars, ed. W. Sieber, \& R. Wielebinski (Dordrecht: Reidel), 115

Cordes, J. M., \& Hankins, T. H. 1977, ApJ, 218, 484

Cordes, J. M., Weisberg, J. M., \& Hankins, T. H. 1990, AJ, 100, 1882 
Ferguson, D. C. 1977, A\&A, 64, 27

Ferguson, D. C., Graham, D. A., Jones, B. B., Seiradakis, J. H., \& Wielebinski, R. 1976, Nature, 260, 25

Ferguson, D. C., \& Seiradakis, J. H. 1978, A\&A, 54, 355

Gangadhara, R. T., Krishan, V., \& Shukla, P. K. 1993, MNRAS, 262, 151

Hankins, T. H. 1971, ApJ, 169, 487

Hankins, T. H. 1972, ApJ, 177, L11

Hankins, T. H. 1996, in Pulsars: Problems and Progress, ed. S. Johnston, M. A. Walker, \& M. Bailes (San Francisco: ASP), ASP Conf. Ser., 105, 197

Hankins, T. H. 2000, in Pulsar Astronomy - 2000 and beyond, ed. M. Kramer, N. Wex, \& R. Wielebinski (San Francisco: ASP), ASP Conf. Ser., 202, 165

Hankins, T. H., \& Boriakoff, V. 1978, Nature, 276, 45

Hankins, T. H., \& Rickett, B. J. 1975, in Methods in Computational Physics: Advances in Research and Applications, vol. 14: Radio Astronomy, ed. B. Alder, S. Fernbach, \& M. Rotenberg (New York: Academic Press, Inc.), 55

Hansen, C. J., \& Cioffi, D. F. 1980, ApJ, 238, 740

Jenet, F. A., \& Anderson, S. B. 1998, PASP, 110, 1467

Kardashev, N. S., Kuzmin, A. D., Nikolaev, N. Y., et al. 1978, SvA, 22, 583, transl. from: AZh, 1978, 55, 1024

Kempner, J. C., Stinebring, D. R., Bailes, M., et al. 1997, Amer. Astron. Soc. Meet., 191, No. 111.19

Kramer, M. 1994, A\&AS, 107, 527

Lange, C., Kramer, M., Wielebinski, R., \& Jessner, A. 1998, A\&A, 332,111

Lyne, A. G., \& Manchester, R. N. 1988, MNRAS, 234, 477

Lyutikov, M., \& Parikh, A. 2000, ApJ, 541, 1016

Manchester, R. N. 1972, ApJ, 172, 43

McDermott, P. N., van Horn, H. M., \& Hansen, C. J. 1988, ApJ, 325, 725
Melrose, D. B. 1992, in Proc. of IAU Colloq. 128, The Magnetospheric Structure and Emission Mechanisms of Radio Pulsars, ed. T. H. Hankins, J. M. Rankin, \& J. A. Gil (Zielona Gora: Pedagogical Univ. Press), 306

Onishchenko, O. G. 1990, in Proc. of the joint Varenna-AbastumaniESA-Nagoya-Potsdam Workshop on Plasma Astrophysics, ESA SP-311, 251

Phillips, J. A., \& Wolszczan, A. 1992, ApJ, 385, 273

Popov, M. V., Smirnova, T. V., \& Soglasnov, V. A. 1987, SvA, 31, 529, transl. from: AZh, 1987, 64, 1013

Rankin, J. M. 1983, ApJ, 274, 333

Rankin, 1993, ApJ, 405, 285

Rickett, B. J. 1975, ApJ, 197, 185

Ruderman, M. A., \& Sutherland, P. G. 1975, ApJ, 196, 51

Sallmen, S., Backer, D. C., Hankins, T. H., Moffett, D. A., \& Lundgren, S. 1999, ApJ, 517, 460

Smirnova, T. V., Soglasnov, V. A., Popov, M. V., \& Novikov, A. Y. 1986, SvA, 30, 51, transl. from: AZh, 1986, 63, 84

Smirnova, T. V., Tul'bashev, S. A., \& Boriakoff, V. 1994, A\&A, 286, 807

Soglasnov, V. A., Popov, M. V., \& Kuzmin, O. A. 1983, SvA, 27, 169, transl. from: AZh, 1983, 60, 293

Soglasnov, V. A., Smirnova, T. V., Popov, M. V., \& Kuzmin, A. D. 1981, SvA, 25, 442, transl. from: AZh, 1981, 58, 771

Taylor, J. H., Manchester, R. N., \& Huguenin, G. R. 1975, ApJ, 195, 513

Thompson, A. R., Moran, J. M., Swenson Jr., G. W., \& Schilizzi, R. T. 1988, Space Sci. Rev., 46, 379

van Horn, H. M. 1980, ApJ, 236, 899

Weatherall, J. C. 1998, ApJ, 506, 341

Wietfeldt, R. D., van Straten, W., Del Rizzo, D., et al. 1998, A\&AS, 131,549 